\title{
Electron pairing in the quantum Hall regime due to neutralon exchange
}

\author{
Giovanni A. Frigeri $\oplus^{1,2}$ and Bernd Rosenow ${ }^{2}$ \\ ${ }^{1}$ Max Planck Institute for Mathematics in the Sciences, D-04103, Leipzig, Germany \\ ${ }^{2}$ Institut für Theoretische Physik, Universität Leipzig, D-04103, Leipzig, Germany
}

(Received 14 July 2020; revised 28 October 2020; accepted 18 November 2020; published 21 December 2020)

\begin{abstract}
The behavior of electrons in condensed matter systems is mostly determined by the repulsive Coulomb interaction. However, under special circumstances, the Coulomb interaction can be effectively attractive, giving rise to electron pairing in unconventional superconductors and specifically designed mesoscopic setups. In quantum Hall systems, electron interactions can play a particularly important role due to the huge degeneracy of Landau levels, leading, for instance, to the emergence of quasiparticles with fractional charge and anyonic statistics. Quantum Hall Fabry-Pérot interferometers (FPI) have attracted increasing attention due to their ability to probe such exotic physics. In addition, such interferometers are affected by electron interactions themselves in interesting ways. Recently, experimental evidence for electron pairing in a quantum Hall FPI was found [Choi et al., Nat. Commun. 6, 7435 (2015)]. Theoretically describing an FPI in the limit of strong backscattering and under the influence of a screened Coulomb interaction, we compute electron shot noise and indeed find a two-fold enhanced Fano factor for some parameters, indicative of electron pairing. This result is explained in terms of an electron interaction due to exchange of neutral interedge plasmons, so-called neutralons.
\end{abstract}

DOI: 10.1103/PhysRevResearch.2.043396

\section{INTRODUCTION}

The quantum Hall $(\mathrm{QH})$ effect is one of the most fascinating phenomena in modern condensed matter physics. It is believed that in the fractional case the elementary excitations have exotic statistics [1-3], such that under the spatial exchange of two quasiparticles the wave function picks up a phase factor that is different from \pm 1 for bosons and fermions. Interferometry is a promising tool for detecting such anyonic statistics in the quantum Hall regime [3,4]. Therefore, the investigation of quantum Hall interferometers has been an active research field recently [5-22]. QH Fabry-Pérot interferometers (FPIs) consist of a Hall bar with two quantum point contacts (QPCs) [4], which introduce a backscattering amplitude between the counterpropagating edge modes. In the limit where backscattering at the QPCs becomes strong, the FPI turns into a weakly coupled quantum dot in the $\mathrm{QH}$ regime. In addition to their potential for revealing fractional [17] and even non-Abelian statistics [23], quantum Hall interferometers turned out to be an amazing tool for exploring the role of interactions [9-16,18-20,22,24].

Interestingly, indications for electron pairing have been observed in a Fabry-Pérot interferometer (FPI) in the integer quantum Hall regime [12]. In particular, it has been observed that the Aharonov-Bohm conductance oscillations have the

Published by the American Physical Society under the terms of the Creative Commons Attribution 4.0 International license. Further distribution of this work must maintain attribution to the author(s) and the published article's title, journal citation, and DOI. Open access publication funded by the Max Planck Society. magnetic flux periodicity equal to half the magnetic flux quantum $h / 2 e$ for bulk filling factors $2<v<5[12,15]$, indicating that interference may be due to charge $2 e$ particles. Besides the halving of the periodicity, shot-noise measurements revealed an interfering charge equal to twice the electron charge $e^{*}=2 e$ [12]. These observations have led the authors of Ref. [12] to suggest the formation of an electron pair in the interfering edge in order to explain the experimental findings. On the theoretical side, the halving of the magnetic flux periodicity of the conductance has later been explained by considering a model with strong edge-edge interaction and a weak bulk-edge interaction [22]. However, no connection between the halved flux period and electron pairing was found in that model [22]. Therefore, it is undoubtedly interesting to further investigate the electronic transport in an interacting FPI in the integer quantum Hall regime and this is the main purpose of this work.

Interactions between particles can be divided into two groups: repulsive and attractive ones. The Coulomb interaction between electrons is known to be repulsive. However, in a variety of systems with electronic degrees of freedom, it has been found that an effective attraction between electrons arises, contrary to naive expectations [25-29]. It is likely that high-temperature superconductivity can be achieved via an effective attractive interaction mediated by Coulomb repulsion [25]. However, the physics of high-temperature superconductors is rather complex, and hence it is valuable to study the possibility of electron attraction in different and simpler systems. For example, effective attraction between electrons due to Coulomb repulsion has been observed in quantum devices made of pristine carbon nanotubes [27] and in a triple quantum dot [28], in which the strong repulsion between two subsystems was exploited to make favorable 


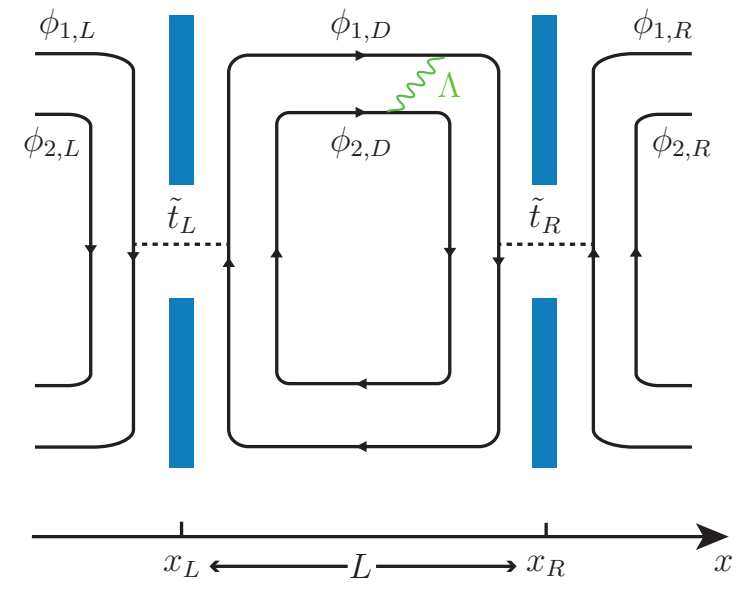

FIG. 1. Schematic view of an electronic Fabry-Pérot interferometer in the closed limit, which is described by a weakly coupled quantum dot in the $\mathrm{QH}$ regime. The quantum point contacts (blue bars) are located at $x_{L}$ and $x_{R}$, respectively, and the dot has size $L$. Electron tunneling (dashed line) occurs only into the outermost edge mode (edge 1), with amplitude $\tilde{t}_{L / R}$. The two edge modes interact in the dot region (wavy line). The parameter $\Lambda$ describes the strength of the edge-edge coupling.

the attraction between the electrons within a given subsystem. Here, we propose a nonequilibrium mechanism for electron-pairing mediated by repulsive Coulomb interactions, occurring in a FPI in the integer quantum Hall regime.

In this work, we consider an FPI in the presence of interedge repulsive interactions and in the strong backscattering limit, i.e., a quantum dot in the $\mathrm{QH}$ regime (see Fig. 1). We choose to focus on this limit because a well-established formalism is available to calculate shot noise [30-34]. On the other hand, it is known that the limit of strong backscattering is intimately related to lowest order interference [16,35], such that this limit provides insight into the behavior of more open interferometers as well. We find that the Fano factor for strongly repulsive interedge interactions is enhanced with respect to the Fano factor of a noninteracting interferometer. At the basis of this enhancement is the participation of neutral interedge plasmon excitations (neutralons) in electron transport. We interpret the enhancement of the Fano factor in terms of a dynamical attraction between electrons taking place in the interfering edge via the exchange of neutralons. We would like to note that an enhanced Fano factor due to internal degrees of freedom was also predicted for backscattering in helical edge states $[36,37]$.

We display in Fig. 2 the magnetic flux averaged value of the Fano factor $F$ as a function of the relative interedge interaction strength $0 \leqslant \Lambda \leqslant 0.9$, where $\Lambda=1$ is the maximum interaction strength allowed by the requirement of electrostatic stability. We see that the enhancement gets stronger when increasing the coupling strength $\Lambda$, which can be understood as being due to the reduction $E_{\sigma}=(1-\Lambda) E_{C}$ of the minimum neutralon excitation energy relative to the charging energy $E_{C}$. Hence, for large $\Lambda$, it is energetically easier to excite neutralons. Additionally, the enhancement of the Fano factor is not a thermal effect, since higher temperatures do not lead

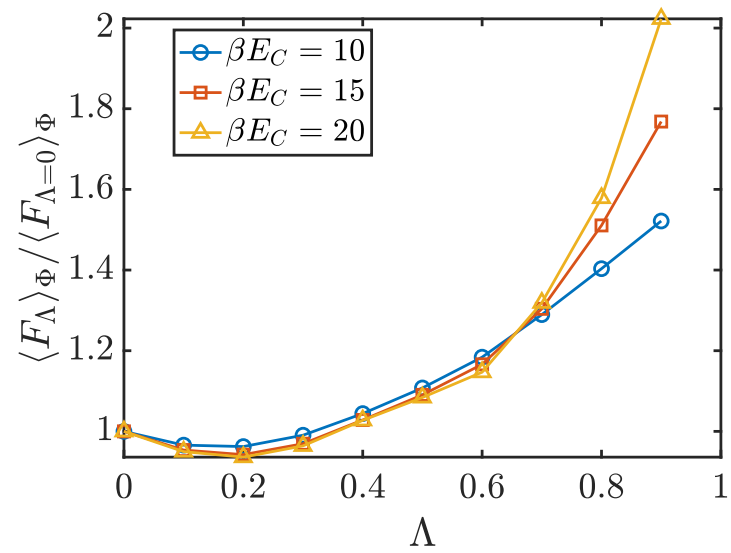

FIG. 2. Ratio between the flux-averaged Fano factor $\left\langle F_{\Lambda}\right\rangle_{\Phi}$ in the presence of interaction and the noninteracting case $\left\langle F_{\Lambda=0}\right\rangle_{\Phi}$ as a function of $\Lambda$ for three different temperatures $\left(\beta E_{C}=10,15,20\right)$. The bias voltage is fixed to $\mathrm{eV} / E_{C}=0.5$, where $E_{c}$ denotes the charging energy of the interferometer.

to a larger Fano factor. Therefore, we can conclude that the enhancement is a genuine nonequilibrium effect.

Electrons can move from the left lead to the right lead of the quantum dot Fig. 1 either (i) independently of each other or (ii) in a correlated way in the sense that one electron leaves the quantum dot in an excited state and a subsequent electron absorbs the excitation to make entering the dot easier. In case (i), the sequence between states is

$$
\left|0,\{0\}_{\sigma}\right\rangle \rightarrow\left|1,\{0\}_{\sigma}\right\rangle \rightarrow\left|0,\{0\}_{\sigma}\right\rangle \rightarrow\left|1,\{0\}_{\sigma}\right\rangle \rightarrow\left|0,\{0\}_{\sigma}\right\rangle .
$$

Here, for a state $\left|n,\{m\}_{\sigma}\right\rangle, n$ denotes the number of additional electrons in the dot, and $m$ denotes the occupancy of the energetically lowest neutralon excitation. In case (ii), a neutralon takes part in the electronic transport. As before, one electron tunnels through the left QPC into the dot. However, a neutralon is now created in the dot when the electron exits. A subsequent electron tunnels more easily into the dot by absorbing the neutralon and then exits the dot without leaving behind an excitation. Accordingly, we are led to consider the sequence of transitions

$$
\left|0,\{0\}_{\sigma}\right\rangle \rightarrow\left|1,\{0\}_{\sigma}\right\rangle \rightarrow\left|0,\{1\}_{\sigma}\right\rangle \rightarrow\left|1,\{0\}_{\sigma}\right\rangle \rightarrow\left|0,\{0\}_{\sigma}\right\rangle .
$$

In order to understand the relative contribution of processes (i) and (ii) to transport current and excess noise, we compute the relative probability of the two processes for a special choice of parameters $\Lambda=0.6, \beta E_{C}=20, \mathrm{eV} / E_{C}=0.5$, and $\Phi=0$. For these parameters, a model taking into account only the three states $\left|0,\{0\}_{\sigma}\right\rangle,\left|0,\{1\}_{\sigma}\right\rangle$, and $\left|1,\{0\}_{\sigma}\right\rangle$ describes the Fano factor with good accuracy; see Fig. 3. The transition rates in processes (i) and (ii) are displayed in Fig. 4.

We see that when the system is in state $\left|1,\{0\}_{\sigma}\right\rangle$ and the electron leaves the dot, the system reaches the state $\left|0,\{0\}_{\sigma}\right\rangle$ with a rate that is almost twice as large as the transition rate to $\left|0,\{1\}_{\sigma}\right\rangle$. However, subsequently the rate for a second electron to enter the dot is almost two orders of magnitude larger for the state $\left|0,\{1\}_{\sigma}\right\rangle$ as compared to the state $\left|0,\{0\}_{\sigma}\right\rangle$ without a neutralon. This big difference is due to the fact that the neutralon provides the necessary energy for a lead electron 


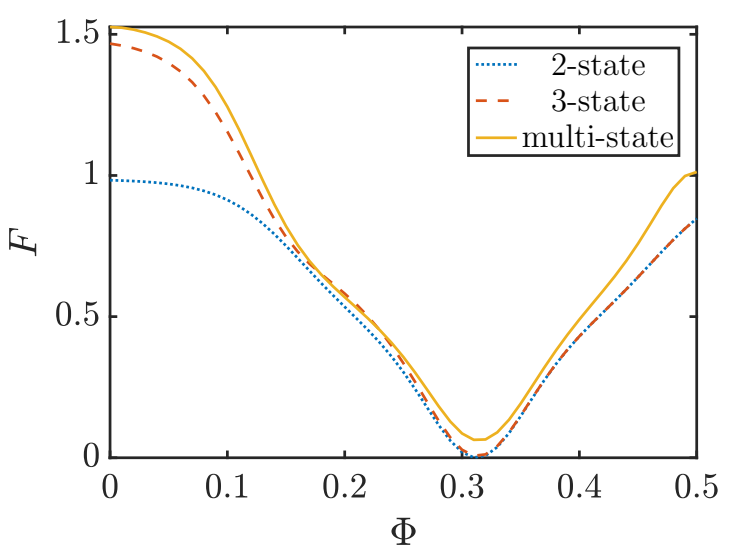

FIG. 3. Fano factor as a function of the magnetic flux for twostate, three-state, and multistate models at $\Lambda=0.6, \beta E_{c}=20$, and $\mathrm{eV} / E_{C}=0.5$. The Fano factor of the system is well represented by the three-state model.

to enter the dot level in the first case, whereas only few electrons in the tail of the Fermi distribution have an energy sufficiently large for entering the dot in the second case. As a consequence, in the case with a neutralon excitation present in the dot, the second electron tunnels through the dot almost instantaneously, such that the tunneling of two electrons takes approximately the same time as the tunneling of a single electron in the case without a neutralon excitation.

When neglecting correlated tunneling of three and more electrons (justified by extra factors of $1 / 2$ for each additional electron tunneling in a correlated fashion), we can adopt an effective description in which either single electrons can tunnel with probability $p_{\text {single }}$ or pairs of electrons with probability $p_{\text {pair }}$. Due to the ratio of transition rates discussed above, we know that $p_{\text {single }}=2 p_{\text {pair }}$, and taking into account the sum rule $p_{\text {single }}+p_{\text {pair }}=1$ we find the values $p_{\text {single }}=2 / 3$ and $p_{\text {pair }}=1 / 3$.

When evaluating the Fano factor for a stochastic process describing tunneling of single electrons and pairs (for details, see Sec. V), we find that the Fano factor is an increasing function of $p_{\text {pair. }}$. In the limit where only single-electron tunneling is possible $\left(p_{\text {pair }}=0\right)$, we obtain the Fano factor for a Poisson process, $F=1$. On the other hand, we obtain $F=2$ in the opposite limit when $p_{\text {pair }}=1$ because only electron pairs tunnel now and we have a Poisson process but with charge carrier $e^{*}=2 e$. The Fano factor takes intermediate values, $1<F<2$, when tunnelings of single electrons and electron pairs coexist. In particular, for the parameters discussed above, we obtain from the stochastic model described

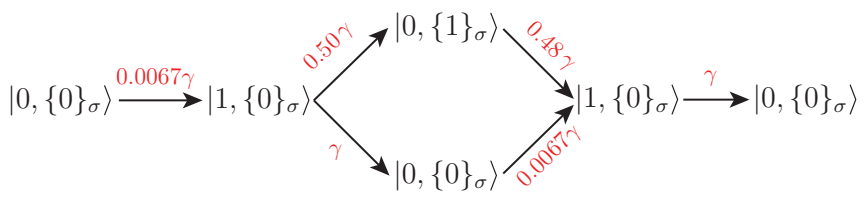

FIG. 4. The two different transport channels when the neutral plasmon excitation is involved. We report the states taking part into the transport of electrons and we indicate on the arrows the respective transition rates at $\Lambda=0.6, \Phi=0, \beta E_{c}=20$, and $\mathrm{eV} / E_{C}=0.5$. in Sec. V $F=1.5$ (red star in Fig. 9). In conclusion, this example gives insight into the mechanism responsible for the enhancement of the Fano factor: A neutralon excitation left behind by one electron makes it easier for a second electron to enter the dot, and thus gives rise to correlated tunneling, comparable to pairing of electrons due to a retarded interaction. We emphasize that for even larger values of $\Lambda>0.6$, correlated processes involving larger number of electrons and neutralons need to be taken into account and can lead to a Fano factor in excess of 2 even when $p_{\text {pair }}<1$.

The remainder of the paper is organized as follows: In Sec. II, we introduce the model used to describe the closed limit of an FPI with repulsive interedge coupling. In Sec. III, we set up the master equation to understand the effect of interactions on the properties of the FPI. The results for the conductance, the noise, and the Fano factor in the presence of strong edge-edge interaction are discussed in Sec. IV. We compare in Sec. V our results with a model in which the tunneling of single electrons and pairs is possible. Finally, we discuss in Sec. VI how the results for an interferometer in the closed limit obtained via the master equation are related to the case of more open interferometers described by a scattering formalism, and comment on the difficulty of extracting an effective charge for interferometers in the closed limit.

\section{MODEL}

In the following, we describe a model which allows us to study how shot noise is influenced by the presence of repulsive Coulomb interactions. We consider an FPI in the closed limit, consisting of two edge modes (filling factor $v=2$ ) coupled by a local repulsive interaction among each other. The interferometer is composed of left $(L)$ and right $(R)$ semi-infinite leads and a finite-size central dot $(D)$ region. Electron tunneling from the leads to the dot, and vice versa, occurs only into the outermost edge mode. We assume that the two edges in the dot region enclose the same magnetic flux $\Phi=A B / \phi_{0}$, where $A$ is the area of the interferometer, $B$ is the perpendicular magnetic field, and $\phi_{0}=h / e$ is the magnetic flux quantum. We are interested in the case of a strongly screened bulk-edge interaction $[12,15,22]$, which allows us to neglect the bulk of the system in the following. The setup is depicted in Fig. 1, and we focus on the consequences of an edge-edge interaction on transport properties. The $L / R$ lead is at equilibrium with the respective reservoir, described by temperature $T$ and voltage $V_{L / R}$. We characterize the $L / R$ reservoir with the respective Fermi function

$$
f_{L / R}(E)=\frac{1}{1+\exp \left[\left(E-\mathrm{eV}_{L / R}\right) / k_{B} T\right]} .
$$

A voltage bias $V=V_{L}-V_{R}$ is applied between the reservoirs, driving the system out of equilibrium.

The left and right leads are semi-infinite, and since an interedge interaction in the lead region would only renormalize tunneling matrix elements, we neglect it here. Then, the Hamiltonian for the $l=L, R$ lead is $(\hbar=1)[38,39]$

$$
H_{l}=\frac{v}{4 \pi} \int_{0}^{\infty} d x\left[\left(\partial_{x} \phi_{1, l}\right)^{2}+\left(\partial_{x} \phi_{2, l}\right)^{2}\right],
$$


where we assumed that the edge modes have the same velocity $v$ and $\phi_{1 / 2, l}$ are the bosonic fields describing edge modes in the $l$ lead which are coupled or uncoupled to the dot region. The fields $\phi_{j, l}(j=1,2)$ satisfy commutation relations [38,39]

$$
\left[\phi_{j, l}(x), \phi_{j, l}\left(x^{\prime}\right)\right]=i \pi \operatorname{sgn}\left(x-x^{\prime}\right)
$$

and are related to one-dimensional electron densities via [38,39]

$$
\rho_{j, l}(x)=\frac{\partial_{x} \phi_{j, l}(x)}{2 \pi} .
$$

On the other hand, a local repulsive interaction couples the edge modes inside the dot region. The appropriate Hamiltonian $H_{D}$ describing the finite-size dot is

$$
\begin{aligned}
H_{D}= & \frac{v}{4 \pi} \int_{0}^{L} d x\left[\left(\partial_{x} \phi_{1, D}\right)^{2}+\left(\partial_{x} \phi_{2, D}\right)^{2}\right. \\
& \left.+2 \Lambda\left(\partial_{x} \phi_{1, D}\right)\left(\partial_{x} \phi_{2, D}\right)\right],
\end{aligned}
$$

where $L$ is the size of the dot, $\phi_{1 / 2, D}$ are the bosonic fields associated with the outer and inner edge modes in the dot, and $\Lambda$ describes the relative strength of the edge-edge coupling $(0 \leqslant \Lambda \leqslant 1)$. Indeed, $\Lambda$ depends on the spatial distance between the edge channels relative to the width of the edge channels, with smaller $\Lambda$ for edges further apart from each other. In principle, the distance between edge states would be larger for smoother confinement [40]. However, there may be additional edge reconstruction for too smooth a confinement, and also the screening length of the Coulomb interaction may increase if, for instance, the setback distance of the two-dimensional electron gas is increased in order to make the confinement smoother, such that it is unclear whether the interedge coupling $\Lambda$ will be reduced in this way. Thus, it may be difficult to tune the parameter $\Lambda$ experimentally. The fields $\phi_{1 / 2, D}$ satisfy the commutation relation Eq. (5) and are related to the electron density via Eq. (6). In order to diagonalize the Hamiltonian Eq. (7), we perform a change of basis

$$
\left(\begin{array}{l}
\phi_{\rho} \\
\phi_{\sigma}
\end{array}\right)=\frac{1}{\sqrt{2}}\left(\begin{array}{cc}
1 & 1 \\
-1 & 1
\end{array}\right)\left(\begin{array}{l}
\phi_{1, D} \\
\phi_{2, D}
\end{array}\right)
$$

allowing us to rewrite Eq. (7) in diagonal form as

$$
H_{D}=\frac{v}{4 \pi} \int_{0}^{L} d x\left[(1+\Lambda)\left(\partial_{x} \phi_{\rho}\right)^{2}+(1-\Lambda)\left(\partial_{x} \phi_{\sigma}\right)^{2}\right]
$$

The fields $\phi_{\rho / \sigma}$ are decomposed into a part $\phi_{\rho / \sigma}^{p}$ obeying periodic boundary conditions, and a nonperiodic part zero mode part $\phi_{\rho / \sigma}^{0}$, which is needed to obtain the correct commutation relations Eq. (5) in a finite-size system [41]:

$$
\phi_{\rho / \sigma}(x)=\phi_{\rho / \sigma}^{p}(x)+\phi_{\rho / \sigma}^{0}(x) .
$$

The periodic part $\phi_{\rho / \sigma}^{p}$ can be expanded in terms of bosonic operators $b_{k, \rho / \sigma}, b_{k, \rho / \sigma}^{\dagger}$, that annihilate or create a charge or neutral plasmon with momentum $k=2 \pi m / L(m=1,2, \ldots)$

$$
\phi_{\rho / \sigma}^{p}(x)=\sum_{k>0} \sqrt{\frac{2 \pi}{k L}} e^{-k \alpha / 2}\left(b_{k, \rho / \sigma} e^{i k x}+\text { H.c. }\right),
$$

where $\alpha$ is a short-distance cutoff. The zero-mode part $\phi_{\rho / \sigma}^{0}$ can be expressed as

$$
\phi_{\rho / \sigma}^{0}(x)=\frac{2 \pi}{L} N_{\rho / \sigma} x-\chi_{\rho, \sigma},
$$

where $N_{\rho / \sigma}$ is the number operator for the charge or neutral sector respectively, and $\chi_{\rho, \sigma}$ is an Hermitian operator canonically conjugate to $N_{\rho / \sigma}$, such that $\left[\chi_{\rho, \sigma}, N_{\rho / \sigma}\right]=i$. In this way, the Hamiltonian in Eq. (7) takes the form

$$
H_{D}=\sum_{\eta=\rho, \sigma}\left(\frac{E_{\eta}}{2} N_{\eta}^{2}+\sum_{m=1}^{+\infty} m E_{\eta} b_{m, \eta}^{\dagger} b_{m, \eta}\right),
$$

where we used the momentum quantization $k=2 \pi m / L$, and we defined the energies

$$
E_{\rho / \sigma}=E_{C}(1 \pm \Lambda) .
$$

Here, the charging energy $E_{C}$ is related to the edge velocity and the size of the dot via $E_{C}=2 \pi v / L$. We obtain the final Hamiltonian for the dot by expressing $N_{\rho / \sigma}$ in terms of the number operator of the outer interfering edge $N_{1}$ and the inner noninterfering edge $N_{2}$ with the transformation in Eq. (8), and including the magnetic flux $\Phi$ via the substitution $N_{1 / 2} \rightarrow N_{1 / 2}-\Phi[18,19,41]$

$$
\begin{aligned}
H_{D}= & \frac{E_{\rho}}{4}\left(N_{1}+N_{2}-2 \Phi\right)^{2}+\frac{E_{\sigma}}{4}\left(N_{1}-N_{2}\right)^{2} \\
& +\sum_{m=1}^{+\infty} m\left[E_{\rho} b_{m, \rho}^{\dagger} b_{m, \rho}+E_{\sigma} b_{m, \sigma}^{\dagger} b_{m, \sigma}\right] .
\end{aligned}
$$

Because of the presence of QPCs at positions $x_{L}$ and $x_{R}$, an electron in the outermost edge can tunnel from and to the leads onto and off the dot. The tunneling Hamiltonian is

$$
H_{T}=\sum_{l=L, R}\left[\tilde{t}_{l} \psi_{1, l}^{\dagger}\left(x_{l}\right) \psi_{1, D}\left(x_{l}\right)+\text { H.c. }\right],
$$

such that an electron is annihilated in the outermost edge of the dot and created in the outermost edge of the $l$ lead, with amplitude $\tilde{t}_{l}$. The Hermitian conjugate term describes the inverse process (from the lead to the dot). The total Hamiltonian of the interacting Fabry-Pérot interferometer is then

$$
H=H_{L}+H_{R}+H_{D}+H_{T},
$$

with the individual terms given by (4), (15), and (16).

\section{RATE EQUATION FORMALISM}

In this section, we introduce the formalism with which we calculate the observables relevant for describing the transport properties of an FPI in the closed limit.

\section{A. Tunneling rates}

By inspection of the Hamiltonian Eq. (15) it is clear that we can describe the state of the dot by the quantum numbers

$$
\left|N_{1}, N_{2},\{n\}_{\rho},\{n\}_{\sigma}\right\rangle,
$$

where $N_{1}$ is the number of electrons on the outermost edge, $N_{2}$ is the number of electrons on the inner edge, and $\{n\}_{\rho}=\left\{n_{1, \rho}, n_{2, \rho}, \ldots\right\},\{n\}_{\sigma}=\left\{n_{1, \sigma}, n_{2, \sigma}, \ldots\right\}$ are the 
occupation numbers of the charge and neutral plasmon modes, respectively. Accordingly, the energy of the dot state $\left|N_{1}, N_{2},\{n\}_{\rho},\{n\}_{\sigma}\right\rangle$ is

$$
\begin{aligned}
E\left(N_{1}, N_{2},\{n\}_{\rho},\{n\}_{\sigma}\right) & \\
= & \frac{E_{\rho}}{4}\left(N_{1}+N_{2}-2 \Phi\right)^{2} \\
& +\frac{E_{\sigma}}{4}\left(N_{1}-N_{2}\right)^{2}+\sum_{m=1}^{+\infty} m\left[E_{\rho} n_{m, \rho}+E_{\sigma} n_{m, \sigma}\right],
\end{aligned}
$$

with $E_{\rho / \sigma}$ defined in Eq. (14). Since we want to be in a regime where the electron number on the outer edge is a good quantum number, the tunneling amplitudes $\left|\tilde{t}_{l}\right|$ need to chosen in such a way that the tunneling conductance into or out of the outer edge is much smaller than the conductance quantum $e^{2} / h$. The rate for the transition from an initial state $|i\rangle$ to a final state $|f\rangle$ due to the small perturbation $H_{T}$ can be calculated using Fermi's golden rule [42]

$$
\Gamma(|i\rangle \rightarrow|f\rangle)=2 \pi\left|\left\langle i\left|H_{T}\right| f\right\rangle\right|^{2} \delta\left(E_{f}-E_{i}\right) .
$$

In Eq. (20), $|i\rangle$ and $|f\rangle$ are the states of the total system, i.e., the dot and the leads, and $E_{i}$ and $E_{f}$ the respective energies. By using Eq. (16) with Eq. (20), integrating over the lead states, and taking into account that the electrons in the leads have a thermal distribution, we find that the total tunneling rates for adding or removing an electron to or from the outermost edge mode in the dot through the $l=L, R$ QPC are

$$
\begin{aligned}
\Gamma_{l}\left(N_{1}, N_{2},\{n\}_{\rho},\{n\}_{\sigma} \rightarrow N_{1}+1, N_{2},\left\{n^{\prime}\right\}_{\rho},\left\{n^{\prime}\right\}_{\sigma}\right) \\
=\gamma_{l} M\left(\{n\}_{\rho},\left\{n^{\prime}\right\}_{\rho}\right) M\left(\{n\}_{\sigma},\left\{n^{\prime}\right\}_{\sigma}\right) \\
\times f_{l}\left(\Delta_{+}\left(N_{1}, N_{2},\{n\}_{\rho},\{n\}_{\sigma},\left\{n^{\prime}\right\}_{\rho},\left\{n^{\prime}\right\}_{\sigma}\right)\right), \\
\Gamma_{l}\left(N_{1}, N_{2},\{n\}_{\rho},\{n\}_{\sigma} \rightarrow N_{1}-1, N_{2},\left\{n^{\prime}\right\}_{\rho},\left\{n^{\prime}\right\}_{\sigma}\right) \\
=\gamma_{l} M\left(\{n\}_{\rho},\left\{n^{\prime}\right\}_{\rho}\right) M\left(\{n\}_{\sigma},\{n\}_{\sigma}\right) \\
\times\left[1-f_{l}\left(-\Delta_{-}\left(N_{1}, N_{2},\{n\}_{\rho},\{n\}_{\sigma},\left\{n^{\prime}\right\}_{\rho},\left\{n^{\prime}\right\}_{\sigma}\right)\right)\right],
\end{aligned}
$$

where we defined the bare tunneling rate $\gamma_{l} \equiv 2 \pi\left|\tilde{t}_{l}\right|^{2} \rho_{F} / L$, with density of states $\rho_{F}=1 / 2 \pi \hbar v$, and $f_{l}$ denoting the Fermi function of the $l$ lead in Eq. (3). In Eqs. (21) and (22), $\left|N_{1}, N_{2},\{n\}_{\rho},\{n\}_{\sigma}\right\rangle$ denote the state of the dot alone. The matrix element $M\left(\{n\},\left\{n^{\prime}\right\}\right)$ accounting for the possible excitation of plasmons is

$$
\begin{aligned}
& M\left(\{n\},\left\{n^{\prime}\right\}\right) \\
& =\frac{\prod_{m=1}^{m_{\max }} e^{-1 / 2 m}\left(\frac{1}{2 m}\right)^{\left|n_{m}-n_{m}^{\prime}\right|} \frac{n_{m}^{(<)} !}{n_{m}^{(>) !}}\left[L_{n_{m}^{(<)}}^{\left|n_{m}-n_{m}^{\prime}\right|}\left(\frac{1}{2 m}\right)\right]^{2}}{\prod_{m=1}^{m_{\max }} e^{-1 / 2 m}},
\end{aligned}
$$

where $n_{m}^{(>)}=\max \left(n_{m}, n_{m}^{\prime}\right), n_{m}^{(<)}=\min \left(n_{m}, n_{m}^{\prime}\right), L_{a}^{b}(x)$ are the associated Laguerre polynomials [43], and $m_{\max }$ is an index such that $n_{m}=n_{m}^{\prime}=0$ for every $m>m_{\max }$. We want to remark that generally the value of $m_{\max }$ in Eq. (23) is different for the excitations in the $\sigma$ and $\rho$ sectors. A detailed derivation of Eq. (23) can be found in Appendix A [31,44]. The addition and subtraction energies are obtained from Eq. (19) as

$$
\begin{aligned}
& \Delta_{ \pm}\left(N_{1}, N_{2},\{n\}_{\rho},\{n\}_{\sigma},\left\{n^{\prime}\right\}_{\rho},\left\{n^{\prime}\right\}_{\sigma}\right) \\
& \quad=E\left(N_{1} \pm 1, N_{2},\left\{n^{\prime}\right\}_{\rho},\left\{n^{\prime}\right\}_{\sigma}\right)-E\left(N_{1}, N_{2},\{n\}_{\rho},\{n\}_{\sigma}\right)
\end{aligned}
$$

\section{B. Master equation}

Knowledge of the transition rates for electron tunneling allows us to set up a rate equation to describe the transport properties of the interferometer and to understand the effect of interactions. The time evolution of the probability that the system is in configuration $N_{1}, N_{2},\{n\}_{\rho},\{n\}_{\sigma}$ at time $t$ is governed by the master equation $[31,44,45]$

$$
\begin{aligned}
\frac{d}{d t} p\left(N_{1}, N_{2},\{n\}_{\rho},\{n\}_{\sigma}, t\right)= & \sum_{l=L, R} \sum_{\left\{n^{\prime}\right\}_{\rho},\left\{n^{\prime}\right\}_{\sigma}}\left\{\Gamma_{l}\left(N_{1}+1, N_{2},\left\{n^{\prime}\right\}_{\rho},\left\{n^{\prime}\right\}_{\sigma} \rightarrow N_{1}, N_{2},\{n\}_{\rho},\{n\}_{\sigma}\right) p\left(N_{1}+1, N_{2},\left\{n^{\prime}\right\}_{\rho},\left\{n^{\prime}\right\}_{\sigma}, t\right)\right. \\
& +\Gamma_{l}\left(N_{1}-1, N_{2},\left\{n^{\prime}\right\}_{\rho},\left\{n^{\prime}\right\}_{\sigma} \rightarrow N_{1}, N_{2},\{n\}_{\rho},\{n\}_{\sigma}\right) p\left(N_{1}-1, N_{2},\left\{n^{\prime}\right\}_{\rho},\left\{n^{\prime}\right\}_{\sigma}, t\right) \\
& -\left[\Gamma_{l}\left(N_{1}, N_{2},\{n\}_{\rho},\{n\}_{\sigma} \rightarrow N_{1}+1, N_{2},\left\{n^{\prime}\right\}_{\rho},\left\{n^{\prime}\right\}_{\sigma}\right)\right. \\
& \left.\left.+\Gamma_{l}\left(N_{1}, N_{2},\{n\}_{\rho},\{n\}_{\sigma} \rightarrow N_{1}-1, N_{2},\left\{n^{\prime}\right\}_{\rho},\left\{n^{\prime}\right\}_{\sigma}\right)\right] p\left(N_{1}, N_{2},\{n\}_{\rho},\{n\}_{\sigma}, t\right)\right\},
\end{aligned}
$$

with the transition rates $\Gamma_{l}$ given by Eqs. (21) and (22). In the long time limit, the system reaches a stationary state with probability distribution

$$
\lim _{t \rightarrow \infty} p\left(N_{1}, N_{2},\{n\}_{\rho},\{n\}_{\sigma}, t\right)=p_{\text {st }}\left(N_{1}, N_{2},\{n\}_{\rho},\{n\}_{\sigma}\right) .
$$

The stationary probability distribution satisfies the condition

$$
\sum_{N_{1}} \sum_{\{n\}_{\rho},\{n\}_{\sigma}} p_{\text {st }}\left(N_{1}, N_{2},\{n\}_{\rho},\{n\}_{\sigma}\right)=p_{\text {st }}\left(N_{2}\right) \text {. }
$$

However, we cannot compute $p_{\text {st }}\left(N_{2}\right)$ within our model, because we assume that the tunneling occurs only in the outermost edge mode. In principle, we would need to specify the dynamics of the innermost edge mode and include the matrix elements for changing the value of $N_{2}$ to solve the master equation in Eq. (25). In our model, the innermost edge mode $N_{2}$ is a slow variable and it has no influence on the pairing mechanism here described. In addition, it is connected to a thermal bath and therefore we approximate the stationary probability distribution of $N_{2}$ as

$$
p_{\mathrm{st}}\left(N_{2}\right)=p_{\mathrm{eq}}\left(N_{2}\right),
$$


with the thermal distribution

$$
p_{\mathrm{eq}}\left(N_{2}\right)=\frac{\sum_{N_{1},\{n\}_{\rho}\{n\}_{\sigma}} e^{-\beta E\left(N_{1}, N_{2},\{n\}_{\rho},\{n\}_{\sigma}\right)}}{\sum_{N_{1}, N_{2},\{n\}_{\rho}\{n\}_{\sigma}} e^{-\beta E\left(N_{1}, N_{2},\{n\}_{\rho},\{n\}_{\sigma}\right)}},
$$

with the energy given by Eq. (19) and inverse temperature $\beta=$ $1 / k_{B} T$. Taking into account the assumption in Eq. (28), it is now possible to solve the master equation in Eq. (25).

\section{Matrix formulation of the master equation}

It order to compute the transport current and noise in an efficient way, it is useful to recast the previous equations in a matrix notation [44]. To that end, we collect the state probabilities $p\left(N_{1}, N_{2},\{n\}_{\rho},\{n\}_{\sigma}, t\right)$ into the probability vector $\boldsymbol{p}(t)$

$$
[\boldsymbol{p}(t)]_{\left\{N_{1}, N_{2},\{n\}_{\rho},\{n\}_{\sigma}\right\}}=p\left(N_{1}, N_{2},\{n\}_{\rho},\{n\}_{\sigma}, t\right),
$$

and we define the matrices $\boldsymbol{\Gamma}_{l}^{( \pm)}$and $\boldsymbol{\Gamma}_{l}^{(0)}$

$$
\begin{aligned}
& \left(\boldsymbol{\Gamma}_{l}^{( \pm)}\right)_{\left\{N_{1} \pm 1, N_{2},\left\{n^{\prime}\right\}_{\rho},\left\{n^{\prime}\right\}_{\sigma}\right\} ;\left\{\left\{N_{1}, N_{2},\{n\}_{\rho},\{n\}_{\sigma}\right\}\right.} \\
& =\Gamma_{l}\left(N_{1}, N_{2},\{n\}_{\rho},\{n\}_{\sigma} \rightarrow N_{1} \pm 1, N_{2},\left\{n^{\prime}\right\}_{\rho},\left\{n^{\prime}\right\}_{\sigma}\right) \\
& \left(\boldsymbol{\Gamma}_{l}^{(0)}\right)_{\left\{N_{1}, N_{2},\{n\}_{\rho},\{n\}_{\sigma}\right\} ;\left\{\left\{N_{1}, N N_{2},\{n\}_{\rho},\{n\}_{\sigma}\right\}\right.} \\
& =\sum_{\left\{n^{\prime}\right\}_{\rho},\left\{n^{\prime}\right\}_{\sigma}}\left[\left(\boldsymbol{\Gamma}_{l}^{(+)}\right)_{\left\{N_{1}+1, N_{2},\left\{n^{\prime}\right\}_{\rho},\left\{n^{\prime}\right\}_{\sigma}\right\} ;\left\{\left\{N_{1}, N_{2},\{n\}_{\rho},\{n\}_{\sigma}\right\}\right.}\right. \\
& \left.\quad+\left(\boldsymbol{\Gamma}_{l}^{(-)}\right)_{\left\{N_{1}-1, N_{2},\left\{n^{\prime}\right\}_{\rho},\left\{n^{\prime}\right\}_{\sigma}\right\} ;\left\{N_{1}, N_{2},\{n\}_{\rho},\{n\}_{\sigma}\right\}}\right]
\end{aligned}
$$

with the transition rates given by Eqs. (21) and (22). The column index of the matrices $\boldsymbol{\Gamma}_{l}^{( \pm)}$represents the initial configuration, while the row index represents the final state. The matrix $\Gamma_{l}^{(0)}$ is a diagonal matrix, whose $n$th element is obtained by summing all the elements in the $n$th column of the $\boldsymbol{\Gamma}_{l}^{( \pm)}$matrices.

In this way, the master equation in Eq. (25) can be cast in the compact form

$$
\frac{d}{d t} \boldsymbol{p}(t)=-\boldsymbol{\Gamma} \boldsymbol{p}(t),
$$

with the transition matrix $\Gamma$, generating the temporal evolution, defined as

$$
\boldsymbol{\Gamma} \equiv \sum_{l=L, R}\left(\boldsymbol{\Gamma}_{l}^{(0)}-\boldsymbol{\Gamma}_{l}^{(+)}-\boldsymbol{\Gamma}_{l}^{(-)}\right),
$$

and we require Eq. (28) to hold. The solution to Eq. (33) is

$$
\boldsymbol{p}(t)=e^{-\Gamma\left(t-t_{0}\right)} \boldsymbol{p}\left(t_{0}\right),
$$

and the stationary probability distribution is given by

$$
\boldsymbol{p}_{\mathrm{st}}=\lim _{t \rightarrow \infty} \boldsymbol{p}(t) \text {. }
$$

The matrix $\Gamma$ always has a zero eigenvalue, since summing over the matrix elements of $\boldsymbol{\Gamma}$ in each column yields zero, as can be seen from (31), (32), and (34). The zero eigenvalue determines the nontrivial stationary solution of Eq. (33).

\section{Average current, noise, and Fano factor}

The average current through the left and right QPC at time $t$ is given by

$$
\left\langle I_{L / R}(t)\right\rangle=e \sum_{\substack{N_{1}, N_{2} \\\{n\}_{\rho},\{n\}_{\sigma}}}\left[\left(\boldsymbol{\Gamma}_{L / R}^{(+)}-\boldsymbol{\Gamma}_{L / R}^{(-)}\right) \boldsymbol{p}(t)\right]_{\left\{N_{1}, N_{2},\{n\}_{\rho},\{n\}_{\sigma}\right\}},
$$

and the total average current flowing trough the system is $\langle I(t)\rangle=\left[\left\langle I_{L}(t)\right\rangle-\left\langle I_{R}(t)\right\rangle\right] / 2$. As a consequence of current conservation, the total stationary current $\langle I\rangle$ is related to the stationary current $\left\langle I_{L / R}\right\rangle$ through the left and right QPC, $\langle I\rangle=\left\langle I_{L}\right\rangle=-\left\langle I_{R}\right\rangle$. Accordingly, we can obtain the stationary current $\langle I\rangle$ as

$$
\langle I\rangle=e \sum_{\substack{N_{1}, N_{2} \\\{n\}_{\rho},\{n\}_{\sigma}}}\left[\left(\boldsymbol{\Gamma}_{L}^{(+)}-\boldsymbol{\Gamma}_{L}^{(-)}\right) \boldsymbol{p}_{\mathrm{st}}\right]_{\left\{N_{1}, N_{2},\{n\}_{\rho},\{n\}_{\sigma}\right\}} .
$$

In addition to the average current, we also need an expression for the fluctuations of the current in time in order to obtain the Fano factor. To this end, we define the noise

$$
S=\lim _{t \rightarrow \infty} 2 \int_{-\infty}^{+\infty} d \tau\left[\langle I(t+\tau) I(t)\rangle-\langle I\rangle^{2}\right] .
$$

The noise $S$ can be computed from the correlation function $K_{l m}(\tau)$ between the left and right currents

$$
K_{l m}(\tau)=\lim _{t \rightarrow \infty}\left\langle I_{l}(t+\tau) I_{m}(t)\right\rangle-\left\langle I_{l}\right\rangle\left\langle I_{m}\right\rangle,
$$

which can be expressed for $\tau \geqslant 0$ as [30]

$$
K_{l m}(\tau)=-\left\langle I_{l}\right\rangle\left\langle I_{m}\right\rangle+e^{2} \sum_{\substack{N_{1}, N_{2} \\\{n\}_{\rho},\{n\}_{\sigma}}}\left\{\left[\left(\boldsymbol{\Gamma}_{l}^{(+)}-\boldsymbol{\Gamma}_{l}^{(-)}\right) e^{-\boldsymbol{\Gamma} \tau}\left(\boldsymbol{\Gamma}_{m}^{(+)}-\boldsymbol{\Gamma}_{m}^{(-)}\right)+\delta_{l m} \delta(\tau)\left(\boldsymbol{\Gamma}_{l}^{(+)}+\boldsymbol{\Gamma}_{l}^{(-)}\right)\right] \boldsymbol{p}_{\mathrm{st}}\right\}_{\left\{N_{1}, N_{2},\{n\}_{\rho},\{n\}_{\sigma}\right\}}
$$

and the case $\tau \leqslant 0$ can be obtained by using $K_{l m}(\tau)=$ $K_{m l}(-\tau)$. Finally, the Fano factor $F$ is defined as the ratio between the excess noise $S-S_{\text {eq }}$ and the average current $\langle I\rangle$ obtained from Eq. (38)

$$
F=\frac{S-S_{\mathrm{eq}}}{2 e\langle I\rangle},
$$

where $S_{\mathrm{eq}}=4 k_{B} T G$ is the Nyquist-Johnson noise, evaluated at zero voltage and temperature $T$, related to the conductance $G$ via the fluctuation-dissipation theorem [42]. In the literature, the Fano factor is sometimes defined as the ratio of total noise and Poissonian noise [46], a definition suitable at zero temperature. Experimentally, in many cases the excess noise 


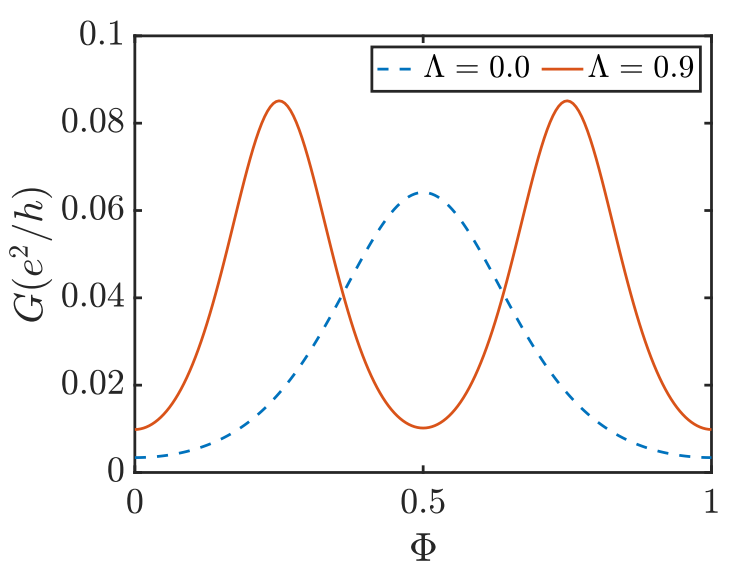

FIG. 5. Conductance as a function of the flux for edge-edge interaction strength $\Lambda=0$ (dashed line) and $\Lambda=0.9$ (solid line). For strong interactions, the magnetic field periodicity is halved, similar to the behavior found for an FPI in the open limit [22]. The temperature is $\beta E_{C}=10$ and $\gamma=0.0513$.

is used to compute the Fano factor [12,47]. In order to make contact with experiments, we perform calculations at finite temperature and use the excess noise instead of the total noise in the definition of the Fano factor, with the advantage that the Fano factor more rapidly approaches its zero temperature limit as a function of bias voltage. In general, a Poissonian Fano factor, $F=1$, indicates that tunneling of subsequent electrons is uncorrelated. The Fano factor is sub-Poissonian, $F<1$, if the electronic transport occurs in an anticorrelated way, while we obtain a super-Poissonian Fano factor, $F>1$, for correlated electron tunneling.

\section{ENHANCEMENT OF THE FANO FACTOR FROM REPULSIVE INTERACTION}

In this section, we are going to show that a many-state model including neutral plasmon excitations is able to capture the features of a strongly interacting interferometer. As a consequence of the nonequilibrium excitation of neutralon modes, the Fano factor is enhanced for a sufficiently strong edge-edge coupling.

In the following, we consider a symmetric interferometer with $\gamma_{L}=\gamma_{R}=\gamma$, and the bias voltage is applied symmetrically $V_{L / R}= \pm V / 2$. We assume that the value of $\gamma$ is independent of the filling factor, and we determine its value by comparing the conductance of a noninteracting FPI $(\Lambda=$ 0 , or equivalently $v=1$ ) with the conductance of a FPI in the coherent tunneling regime (see Sec. VI). However, we would like to point out that ultimately the magnitude $\gamma$ of the tunneling rate is not important because the Fano factor is independent of it, since both the current and the noise are linear in $\gamma$.

Using the rate equation formalism described in the previous section, we first compute the average current and the conductance. In Fig. 5, we plot the conductance as a function of the magnetic flux for $\Lambda=0.0$ and $\Lambda=0.9$. In the absence of interactions, $\Lambda=0$, the system is in the Aharonov-Bohm $\mathrm{AB}$ ) regime $[9,12,13,19,22]$, and the flux periodicity of the conductance is one flux quantum, $\Delta \Phi=1$. The strong edge-

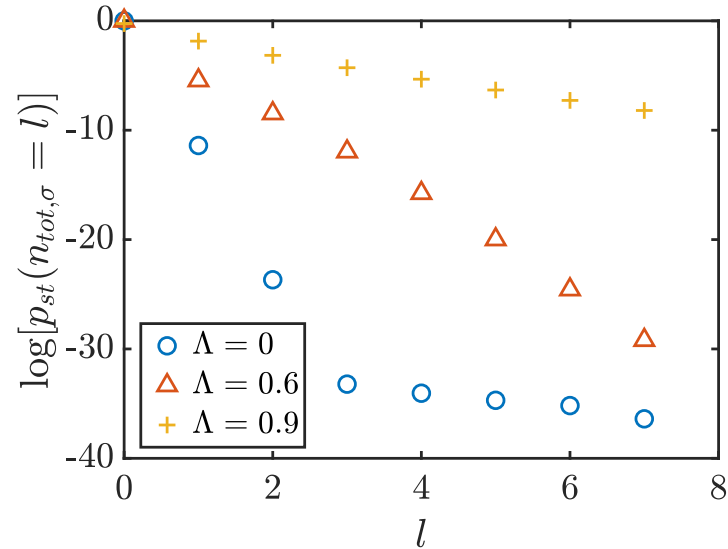

FIG. 6. Probability (in log scale) to have $l$ neutral plasmon excitations in the system, defined in Eq. (44), for different values of the edge-edge interaction strength $\Lambda$. A strong intraedge coupling favors the creation of the neutral plasmon excitations. The magnetic flux is set to $\Phi=0$, the temperature is $\beta E_{C}=20$, and the bias voltage $\mathrm{eV} / E_{C}=0.5$.

edge interaction places the interferometer in the so-called $\mathrm{AB}^{\prime}$ regime $[12,15,22]$, where we observe a conductance with a flux periodicity equal to half the magnetic flux quantum, $\Delta \Phi=1 / 2$. The fact that we obtain the halving of the oscillation period for an interferometer in both the closed and in the open limit [22] is due to the fact that the closed limit conductance can be expressed as a Fourier series in the magnetic flux and that the open limit contributes the leading periodicity in this Fourier expansion. We want to remark that the plasmon excitations are not fundamental to describe magnetic flux dependence of the conductance and that they are not responsible for the period halving, since they do not couple to the magnetic flux as it can be seen from Eq. (19).

However, we will see momentarily that the neutralon excitations are a key ingredient for the enhancement of current fluctuations and the Fano factor. First, we look at the probability of neutral plasmon excitations in the system. Given the neutral plasmons configuration $\{n\}_{\sigma}=\left\{n_{1, \sigma}, n_{2, \sigma}, \ldots\right\}$, we define the total number of neutral plasmon $n_{\mathrm{tot}, \sigma}$ as

$$
n_{\mathrm{tot}, \sigma}=\sum_{m=1}^{\infty} n_{m, \sigma} .
$$

The probability to have $l$ neutral plasmons in the dot is defined as

$$
p_{\mathrm{st}}\left(n_{\mathrm{tot}, \sigma}=l\right)=\sum_{\substack{N_{1}, N_{2} \\\{n\}_{\rho}}} \sum_{\{n\}_{\sigma}}^{\prime} p_{\mathrm{st}}\left(N_{1}, N_{2},\{n\}_{\rho},\{n\}_{\sigma}\right),
$$

where $p_{\mathrm{st}}\left(N_{1}, N_{2},\{n\}_{\rho},\{n\}_{\sigma}\right)$ is obtained from the master equation, and the primed sum is constrained to those neutral plasmons configurations $\{n\}_{\sigma}$ satisfying $n_{\text {tot }, \sigma}=l$. In Fig. 6, we report the probability defined in Eq. (44) for different values of the edge-edge interaction $\Lambda$ at $\Phi=0, \beta E_{C}=20$, and $\mathrm{eV} / E_{C}=0.5$. It is evident that a strong edge-edge coupling makes it easier to create the neutral plasmons. Indeed, a smaller amount of energy is required to excite them because the energy gap for the neutral plasmons $E_{\sigma}$ decreases with 

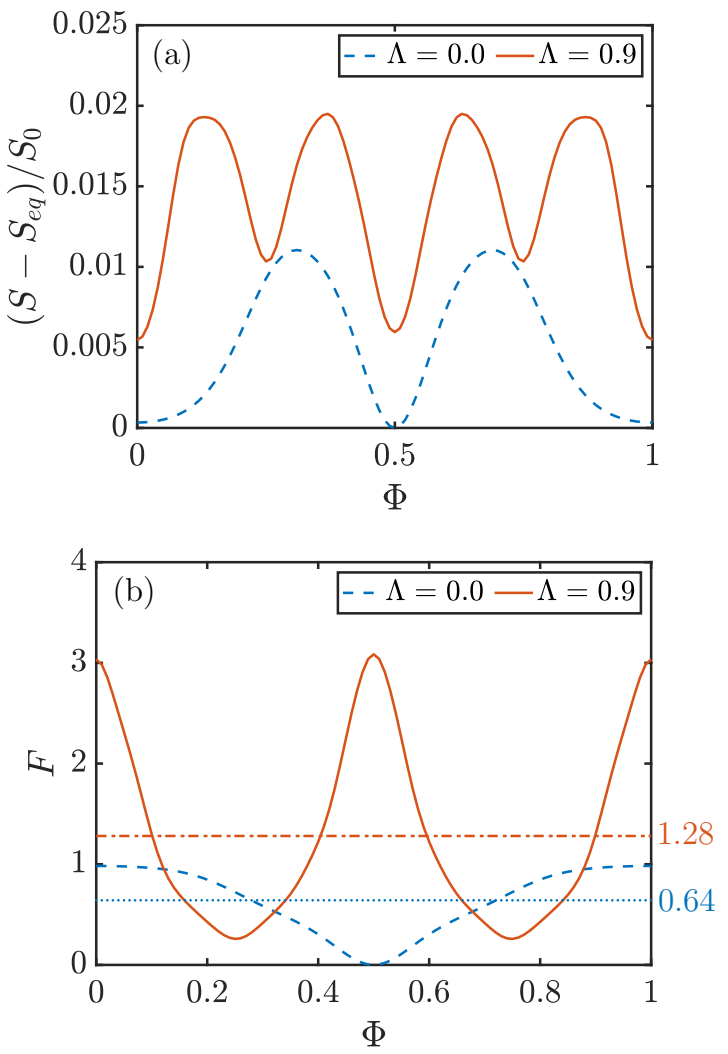

FIG. 7. (a) Excess noise $S-S_{\text {eq }}$, normalized by the reference noise $S_{0}=2 e^{2} E_{C} / h$, as a function of the flux for edge-edge interaction strength $\Lambda=0$ (dashed line) and $\Lambda=0.9$ (solid line). The parameters are $\beta E_{C}=20, \mathrm{eV} / E_{C}=0.5$, and $\gamma=0.0513$. (b) Fano factor $F$ as a function of the flux in the noninteracting limit $(\Lambda=0$, dashed line) and in the strongly interacting limit $(\Lambda=0.9$, solid line). The Fano factor is significantly enhanced in the presence of strong interedge coupling. The dotted (dash-dotted) line represents the average value of the Fano factor for $\Lambda=0(\Lambda=0.9)$, as defined in Eq. (45). We set the temperature $\beta E_{C}=20$ and bias voltage $\mathrm{eV} / E_{C}=0.5$.

the strength of the edge-edge interaction $\Lambda$, as can be seen from Eq. (14). While for an intermediate interaction strength $\Lambda=0.6$ the excitation probability decreases quickly with increasing excitation number $l$, for a large interaction strength $\Lambda=0.9$ a large number of neutral plasmons can be excited, and a many-state model is necessary to correctly describe the properties of a strongly interacting interferometer.

In Fig. 7, we report the excess noise and the Fano factor as a function of the flux for a noninteracting, $\Lambda=0$, and a strongly interacting FPI, $\Lambda=0.9$. The excess noise is an oscillatory function of the flux, and we observe that the current noise is more pronounced for stronger interedge interactions. Indeed, both the maximum and minimum values of the excess noise for $\Lambda=0.9$ are larger than the respective values in the noninteracting case. At $\Lambda=0$, the excess noise vanishes for $\Phi=1 / 2$, since for this value of flux the total noise is entirely due to thermal noise, $S=S_{\text {eq }}$. In contrast, the excess noise never vanishes, $S>S_{\mathrm{eq}}$, in the strongly interacting case, and this is a signature of the participation of the neutral plasmons to the transport. Both the excess noise and the Fano factor have the same flux periodicity as the conductance. By comparing Fig. 5 with Fig. 7(b), we see that when the conductance increases, in general the Fano factor decreases and a maximum or minimum in $F$ corresponds to a minimum or maximum in $G$. An increase of the conductance implies that electrons are more easily transported through the system.

When one electron enters into the dot, it is necessary to wait for that electron to leave the dot before adding another electron, as a consequence of the Pauli exclusion principle. Therefore, the transfer of two subsequent electrons is anticorrelated, and the Fano factor is suppressed with respect to the Poissonian case of noninteracting electrons. However, the transfer of subsequent electrons is almost uncorrelated at $\Phi=$ 0 in the absence of interedge interactions, $\Lambda=0$, because in this case transport is limited by the exponentially suppressed rate for electrons entering the dot in the limit $\beta E_{C} \gg 1$, while electrons leave the dot almost instantaneously. As a consequence, for $\Phi=0$ and $\Lambda=0$ we obtain a Fano factor $F \approx 1$. By increasing $\Phi$, the effect of Pauli blockade becomes more prominent, and this gives a sub-Poissonian Fano factor, $F<1$. At the energetically degenerate point at $\Phi=0.5$, the noise is totally thermal and hence the Fano factor vanishes, $F=0$. On the other hand, the participation of neutralons in electron transport in the strongly interacting limit implies an enhancement of the Fano factor, as can be seen from Fig. 7(b). Indeed, the maximum of $F$ at $\Lambda=0.9$ is three times higher than the maximum of $F$ at $\Lambda=0$, indicating a significant correlation between subsequent tunneling events. In addition, due to the excitation of neutral plasmons implies, the minimum of $F$ occurs at a finite value. Hence, we have two competing mechanisms affecting the Fano factor: The Pauli exclusion principle in combination with the charging energy of the dot tend to reduce it, while an upward correction is caused by the involvement of the neutralons in electron transport. We also indicate in Fig. 7(b) the average value of $F$ with respect to the flux, defined as

$$
\langle F\rangle_{\Phi}=\int_{0}^{1} d \Phi F(\Phi) .
$$

To quantify the enhancement of the Fano factor, we compare in Fig. 8 the Fano factor $F_{\Lambda}$ in the presence of interaction with the noninteracting case $F_{\Lambda=0}$ at $\Phi=0$ for different values of $\Lambda$. As in Fig. 2, the Fano factor at $\Phi=0$ is more strongly enhanced for larger interedge coupling $\Lambda$ and at lower temperature. To summarize, we have seen that a strong edge-edge interaction favors the excitation of neutral plasmons and also leads to an enhancement of the Fano factor. In the next section, we will establish a link between the presence of neutralon excitations and an enhancement of the Fano factor.

\section{ELECTRON ATTRACTION MEDIATED BY NEUTRAL PLASMONS}

In this section, we want to show that it is possible for electrons passing through the interfering edge to attract each other via the exchange of neutral plasmon excitations in the presence of a sufficiently strong repulsive interaction. The formation of electron bunches explains the enhancement of the Fano factor discussed in the previous section. 


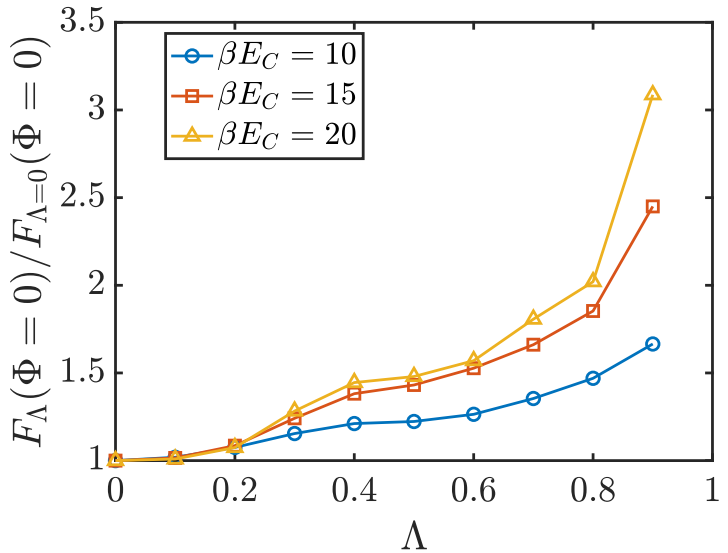

FIG. 8. Ratio between the Fano factor $F_{\Lambda}$ in the presence of interaction and the noninteracting case $F_{\Lambda=0}$ at flux $\Phi=0$ for different values of $\Lambda$ and three different temperatures $\left(\beta E_{C}=10,15,20\right)$. The enhancement of the Fano factor is more pronounced for stronger edge-edge coupling $\Lambda$ and lower temperature.

We now argue that for intermediate values of the interaction it is sufficient to consider a three-state model with charge states $N_{1}=0,1$ and the lowest energy neutral excitation $\{n\}_{\sigma}=\{1,0, \ldots\}_{\sigma} \equiv\{1\}_{\sigma}$, defined by the basis states

$$
\left|0,\{0\}_{\sigma}\right\rangle, \quad\left|1,\{0\}_{\sigma}\right\rangle, \quad\left|0,\{1\}_{\sigma}\right\rangle .
$$

Here, we have a fixed $N_{2}=0$ and do not consider excitation of charged plasmon. In Fig. 3, the Fano factor as obtained from the rate equation formalism is plotted as a function of flux for the interaction strength $\Lambda=0.6$, temperature $\beta E_{C}=20$, and voltage $\mathrm{eV} / E_{C}=0.5$. By comparing the result of a twostate model (without any plasmon excitations), the three-state model defined above, and the full model, we see that it is necessary to take into account plasmon excitations in order to correctly model the properties of an FPI with the chosen parameters. In addition, the three-state model captures a large part of the Fano factor enhancement. Therefore, the most relevant transport channels are the ones described in Eqs. (1) and (2). As explained in Sec. I, the exchange of a neutral plasmon makes the tunneling of subsequent electrons correlated and this produces the enhancement of the Fano factor. We note that the exact Fano factor in Fig. 3 does not yet have the $\mathrm{AB}^{\prime}$ periodicity $\Delta \Phi=1 / 2$. Indeed, we see that at $\Lambda=0.6$ a subleading $\mathrm{AB}$ component with periodicity $\Delta \Phi=1$ is present in the Fourier spectrum of $F$, in addition to the dominant $\mathrm{AB}^{\prime}$ component, and it cannot be neglected [22]. By increasing the value of $\Lambda$, the $\mathrm{AB}$ component becomes less important. However, the three-state model is inapplicable if we increase the value of $\Lambda$, because it becomes easier to excite multiple plasmon states and so a many-state model would be necessary to describe the properties of the interferometer.

Finally, we want to confirm that the enhancement of the Fano factor can be interpreted in terms of single electrons and electron pairs tunneling. The probability that $N_{\mathrm{el}}$ electrons

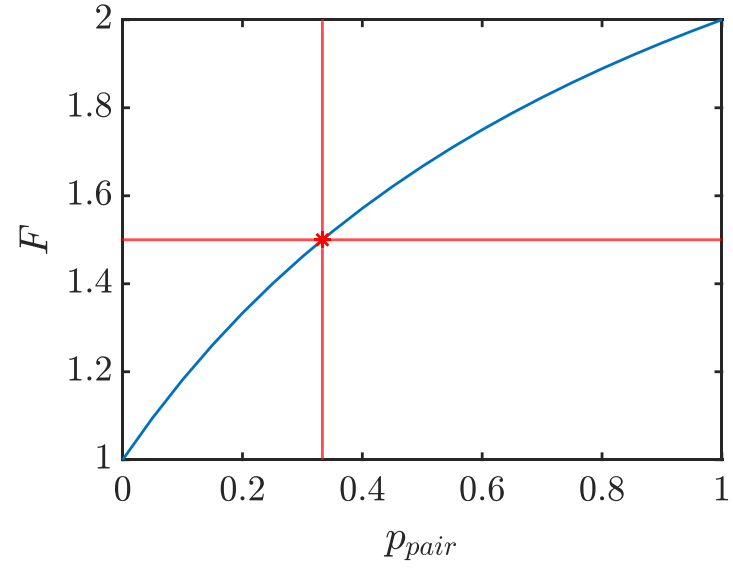

FIG. 9. Fano factor as a function of the probability for an electron pair to tunnel $p_{\text {pair }}$ for the model described by (47), (48), and (50), in which individual electron and pair tunnelings coexist. The point indicated with a red star at $p_{\text {pair }}=p_{\text {single }} / 2=1 / 3$ describes the three-state model in Fig. 3 at $\Phi=0$, as it can be seen by looking at the transition rates in the tree diagram of Fig. 4. The Fano factor at $p_{\text {pair }}=1 / 3$ is $F=1.5$, in very good agreement with the the enhancement found in Fig. 8 at $\Lambda=0.6$.

tunnel through the system is given by

$$
p\left(N_{\mathrm{el}}\right)=\sum_{N_{\text {events }}=N_{\mathrm{el}} / 2}^{N_{\mathrm{el}}} p\left(N_{\mathrm{el}} \mid N_{\mathrm{events}}\right) p\left(N_{\mathrm{events}}\right),
$$

where $N_{\text {events }}$ is the number of tunneling events (either tunneling of single electrons or of electron pairs), $p$ ( $\left.N_{\text {events }}\right)$ is the corresponding probability, and $p\left(N_{\mathrm{el}} \mid N_{\text {events }}\right)$ is the conditional probability for a total of $N_{\mathrm{el}}$ electrons to tunnel, given $N_{\text {events }}$ tunneling events. The lower bound of the summation in Eq. (47) corresponds to the case in which only electron pairs tunnel, while the upper bound corresponds to a situation in which only individual electrons tunnel. We assume that $p\left(N_{\text {events }}\right)$ is given by a Poisson distribution expressed in terms of the average number of tunneling events $\left\langle N_{\text {events }}\right\rangle$

$$
p\left(N_{\text {events }}\right)=\frac{\left\langle N_{\text {events }}\right\rangle^{N_{\text {events }}}}{N_{\text {events }} !} e^{-\left\langle N_{\text {events }}\right\rangle} .
$$

Given $N_{\text {events }}$ tunneling events, we have $k$ single electrons and $\left(N_{\text {events }}-k\right)$ pairs tunneling. Accordingly, the number of tunneled electrons is given by

$$
N_{\mathrm{el}}=k+2\left(N_{\text {events }}-k\right)=2 N_{\text {events }}-k,
$$

and the conditional probability is a binomial distribution

$$
p\left(N_{\text {el }} \mid N_{\text {events }}\right)=\left(\begin{array}{c}
N_{\text {events }} \\
2 N_{\text {events }}-N_{\text {el }}
\end{array}\right) p_{\text {single }}^{2 N_{\text {events }}-N_{\text {el }}} p_{\text {pair }}^{N_{\text {el }}-N_{\text {events }}} .
$$

By combining (47), (48), and (50), we can obtain the average number of tunneled electrons $\left\langle N_{\mathrm{el}}\right\rangle$, the variance $\operatorname{Var}\left(N_{\mathrm{el}}\right)$, and the Fano factor $F=\operatorname{Var}\left(N_{\mathrm{el}}\right) /\left\langle N_{\mathrm{el}}\right\rangle$. In Fig. 9, we plot the Fano factor obtained from (47), (48), and (50) as a function of $p_{\text {pair }}$. In particular, we have $F=1.5$ (red star in Fig. 9) at $p_{\text {pair }}=p_{\text {single }} / 2=1 / 3$, representing the interferometer for the parameters $\Lambda=0.6, \beta E_{C}=20, \mathrm{eV} / E_{C}=0.5$, and $\Phi=$ 0 (compare the transition rates in Fig. 4). This value is in very good agreement with the actual enhancement $F_{\Lambda}(\Phi=$ 
$0) / F_{\Lambda=0}(\Phi=0)=1.57$ found in Fig. 8, implying that our results can indeed be interpreted in terms of single and electron pair tunnelings.

\section{COMPARISON BETWEEN MASTER EQUATION AND SCATTERING FORMALISM}

The goal of this section is twofold. First, we analyze under what conditions results obtained from the master equation formalism are applicable to the description of Fabry-Pérot interferometers with a finite QPC transparency. Second, we explain that in the absence of dephasing it is not possible to extract an effective charge from excess noise computed in the master equation formalism. For this reason, we have presented our results in the preceding section in terms of a relative enhancement of the Fano factor rather than in terms of an effective interfering charge.

A key ingredient in interferometry is phase coherence. Therefore, it is natural to ask under which condition the results from the master equation approach are equivalent to those obtained from a scattering formalism describing coherent tunneling of electrons. To this end, we here compare observables characterizing electronic transport of an FPI at filling factor $v=1$ obtained with the master equation (ME) with results obtained via the scattering formalism (SF) $[46,48,49]$.

The fundamental quantity that we need to compute in the SF is the transmission probability $\mathcal{T}$. In a round trip along the interference cell, the electron picks up the phase

$$
\theta(E)=2 \pi\left(\frac{E}{E_{C}}+\Phi\right),
$$

where the first part is a dynamical phase and the second contribution is the Aharonov-Bohm phase [50]. When summing over all possible numbers of windings around the interference cell, the transmission probability of the FPI is found to be

$$
\mathcal{T}(E)=\frac{\left|t_{L}\right|^{2}\left|t_{R}\right|^{2}}{|1+| r_{L}|| r_{R}\left|e^{2 \pi i\left(E / E_{C}+\Phi\right)}\right|^{2}},
$$

with transmission (reflection) amplitude $t_{l}\left(r_{l}\right)$ at the $l$ th QPC. When deriving Eq. (52), we assumed that $\operatorname{sgn}\left(r_{L} r_{R}\right)=-1$ such that the conductance obtained with the SF has a maximum at $\Phi=0.5$, like the conductance computed with the ME at $\Lambda=0$ (see Fig. 10). In the weak tunneling limit $\left|t_{L}\right|,\left|t_{R}\right| \ll$ 1 , the transmission probability is sharply peaked around the discrete energy levels $E_{n}$, such that $\theta\left(E_{n}\right)=\pi(1+2 n)$, and it can be approximated by the Breit-Wigner formula [51]

$$
\mathcal{T}(E) \simeq \sum_{n} \frac{\Gamma_{\mathrm{FPI}, L} \Gamma_{\mathrm{FPI}, R}}{\left(\Gamma_{\mathrm{FPI}} / 2\right)^{2}+\left(E+E_{C} \Phi-E_{n}\right)^{2}},
$$

with the total width of the resonant level given by

$$
\Gamma_{\mathrm{FPI}}=\Gamma_{\mathrm{FPI}, L}+\Gamma_{\mathrm{FPI}, R},
$$

obtained from the partial widths

$$
\Gamma_{\mathrm{FPI}, L / R}=\frac{E_{C}}{2 \pi}\left|t_{L / R}\right|^{2} .
$$

Approximating the transmission probability Eq. (52) by Eq. (53) becomes exact in the limit $\Gamma_{\mathrm{FPI}} / E_{C} \rightarrow 0$. We can
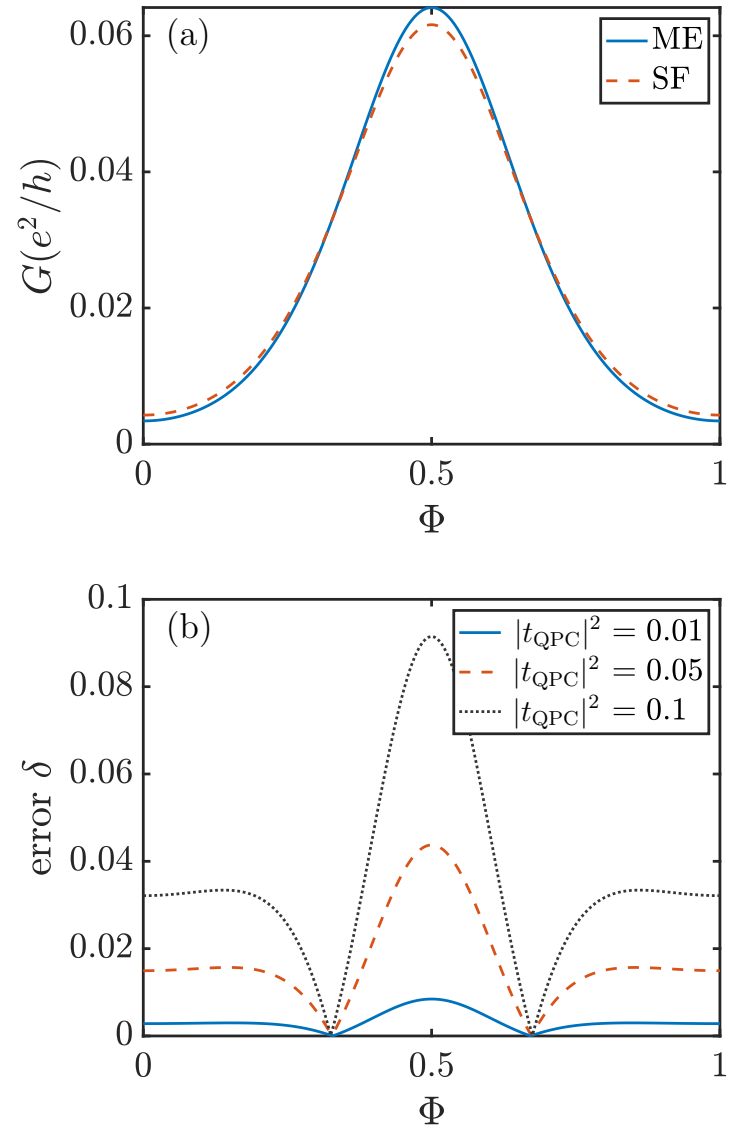

FIG. 10. (a) Conductance at filling factor $v=1$ (equivalent to $\Lambda=0$ ) as a function of the flux calculated from the master equation (ME, solid line) and the scattering formalism (SF, dashed line). The temperature is chosen as $\beta E_{C}=10$, and the QPC transparency is $\left|t_{\mathrm{QPC}}\right|^{2}=0.05$ in the SF. By requiring that the value of the fluxaveraged conductance is the same for the SF and the ME, we can determine the bare tunneling rate entering in the ME to be $h \gamma / E_{C}=$ 0.0513. (b) Error $\delta$, defined in Eq. (60), as a function of the flux at $\beta E_{C}=10$ for different values of the QPC's transparency $\left|t_{\mathrm{QPC}}\right|^{2}$, such that the condition in Eq. (59) is fulfilled. The error decreases when the interferometer is more closed.

obtain from Eq. (52) the current [46]

$$
I=\frac{e}{2 \pi} \int d E \mathcal{T}(E)\left[f_{L}(E)-f_{R}(E)\right],
$$

and subsequently the two terminal conductance

$$
G=\frac{e^{2}}{2 \pi} \int d E \mathcal{T}(E)\left(-\frac{\partial f}{\partial E}\right) .
$$

Finally, the noise is given by [46]

$$
\begin{aligned}
S= & \frac{e^{2}}{\pi} \int d E\left\{\mathcal{T}(E)\left[f_{L}\left(1-f_{L}\right)+f_{R}\left(1-f_{R}\right)\right]\right. \\
& \left.+\mathcal{T}(E)[1-\mathcal{T}(E)]\left(f_{L}-f_{R}\right)^{2}\right\} .
\end{aligned}
$$

The Fano factor is then obtained from Eqs. (56) and (58) and by using the definition Eq. (42).

In the following, we assume the FPI to be symmetric, by setting $\left|t_{L}\right|=\left|t_{R}\right| \equiv\left|t_{\mathrm{QPC}}\right|$ for the SF in Eq. (52). The symmetry of the FPI is reflected in the ME by letting $\gamma_{L}=\gamma_{R} \equiv \gamma$ 
in Eqs. (21) and (22). Moreover, we assume that the voltage bias is applied symmetrically, $V_{L / R}= \pm V / 2$. One important difference between the $\mathrm{SF}$ and the ME is that the intrinsic width of the resonant level is neglected in the ME. In order to compare results between the two formalisms, we hence consider the limit in which the temperature $k_{B} T$ is much bigger than the intrinsic width of the resonant level $\Gamma_{\mathrm{FPI}}$. By using Eqs. (55) and (54) and the symmetry of the FPI, this condition is expressed as

$$
\beta E_{C} \ll \frac{\pi}{\left|t_{\mathrm{QPC}}\right|^{2}} .
$$

In Fig. 10(a), we show the conductance as a function of the flux calculated from the master equation $v=1(\mathrm{ME}$, solid line) and the scattering formalism in Eq. (57) (SF, dashed line), under the condition in Eq. (59). The bare tunneling rate $\gamma$ entering in the $\mathrm{ME}$ is determined by requiring that the average value of the conductance is the same for the ME and the SF. The conductance is periodic in the flux with a period of one flux quantum $\Delta \Phi=1$, as expected from the AharonovBohm effect $[12,19,22]$. We see that at integer values of the flux $\Phi=n \in \mathbb{Z}$ the conductance has a minimum, while it reaches the maximum value when the flux is half-integer $\Phi=n+1 / 2$. Indeed, it costs a maximum amount of energy to add (remove) electrons to (from) the dot when $\Phi=n$ and so the transport is almost blockaded and the conductance is small. On the other hand, it is energetically easy to transport electrons through the system at $\Phi=n+1 / 2$, and for this reason the conductance has a maximum. Good agreement is found between the conductance calculated with the two different approaches under the condition in Eq. (59). To quantify the agreement between the two approaches, we define the error $\delta$ as

$$
\delta=\frac{\left|G_{\mathrm{SF}}-G_{\mathrm{ME}}\right|}{\max \left(G_{\mathrm{SF}}\right)-\min \left(G_{\mathrm{SF}}\right)},
$$

and $G_{\mathrm{SF} / \mathrm{ME}}$ denotes the conductance calculated with the SF or ME. The error is plotted as a function of the flux for different values of the QPC's transparency $\left|t_{\mathrm{QPC}}\right|^{2}$ at fixed temperature $\beta E_{C}=10$ in Fig. $10(\mathrm{~b})$. We see that the error approaches zero when the value of $\left|t_{\mathrm{QPC}}\right|^{2}$ decreases, such that the interferometer is more closed. However, the error does not converge to zero if the temperature is increased while keeping fixed the value of the QPC's transmission $\left|t_{\mathrm{QPC}}\right|^{2}$. Therefore, we can conclude that at least for describing the flux dependence of the conductance one can use the ME to study the transport properties of an FPI in the coherent tunneling regime if (i) the interferometer is sufficiently closed and (ii) such that Eq. (59) is satisfied. Specifically, for a QPC transparency $\left|t_{\mathrm{QPC}}\right|^{2}=0.05$, the maximum error in the conductance is less than $5 \%$.

The next quantity we compute is nonequilibrium noise, as defined in Eq. (39). We plot in Fig. 11 both the excess noise $S-S_{\text {eq }}$ (normalized by the reference noise $S_{0} \equiv 2 e^{2} E_{C} / h$ ) and the Fano factor $F$ as a function of the magnetic flux. The two quantities are calculated with both the ME [solid line, Eq. (41)] and the SF [dashed line, Eq. (58)]. We want to remark that the Fano factor obtained from the ME is independent of $\gamma$, because the noise and the current are both linear in this parameter. We see immediately that the ME and
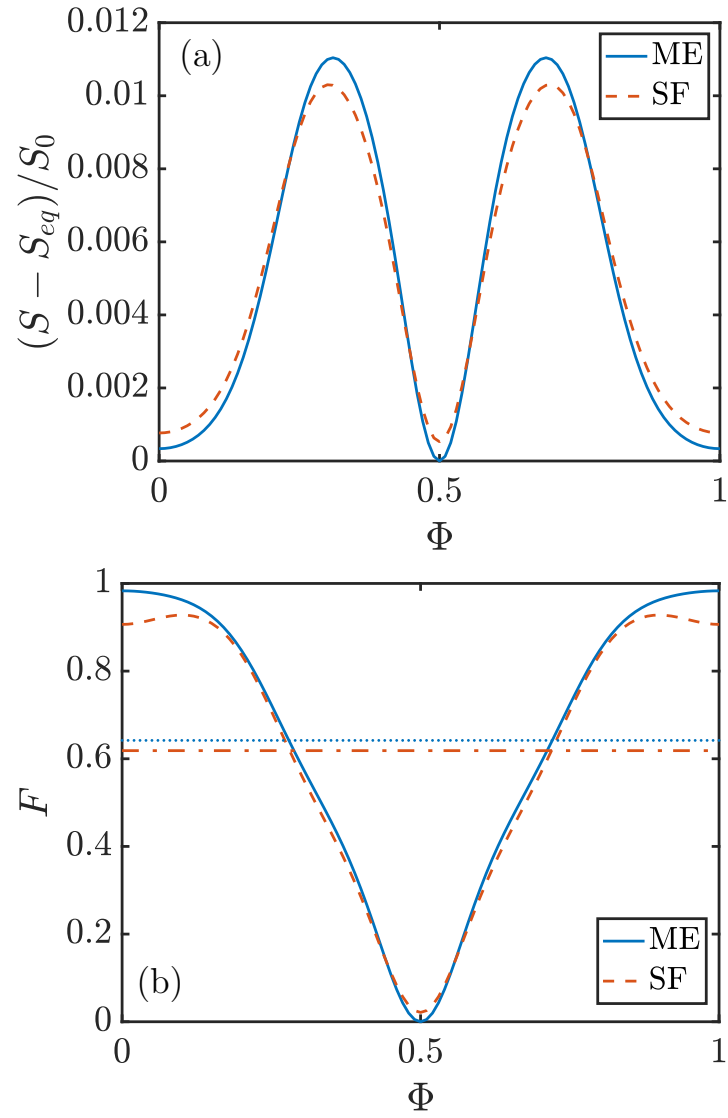

FIG. 11. (a) Excess noise $S-S_{\text {eq }}$ divided by the reference noise $S_{0} \equiv 2 e^{2} E_{C} / h$ at filling factor $v=1$ as a function of the flux $\Phi$ obtained from the master equation (ME) and the scattering formalism (SF). Parameters are chosen as $\beta E_{C}=20$, bias voltage $\mathrm{eV} / E_{C}=0.5$, $\left|t_{\mathrm{QPC}}\right|^{2}=0.05$, and $h \gamma / E_{C}=0.0513$. (b) Fano factor at filling factor $v=1$ as a function of the flux $\Phi$ obtained from the master equation (ME) and the scattering formalism (SF). The dot-dash-dotted line represents the average value of the Fano factor, defined in Eq. (45), for the ME/SF. Parameters are chosen as $\beta E_{C}=20$, bias voltage $\mathrm{eV} / E_{C}=0.5$, and $\left|t_{\mathrm{QPC}}\right|^{2}=0.05$.

the SF give very similar results for the noise and the Fano factor, when $\left|t_{\mathrm{QPC}}\right|^{2} \ll 1$ and when temperature and QPC transparency satisfy Eq. (59).

In the experimental work of Ref. [12], in addition to measuring shot noise, the authors extracted an effective charge $e^{*}$ from shot-noise measurements. From the transmitted current $I$ and the noise $S$, the interfering charge $e^{*}$ was obtained via the formula $[12,52,53]$

$$
\begin{aligned}
S-S_{\text {eq }}= & 2 e^{*} I_{\text {imp }} \mathcal{T}_{\exp }\left(1-\frac{e}{e^{*}} \mathcal{T}_{\exp }\right) \\
& \times\left[\operatorname{coth}\left(\frac{\beta e^{*} V}{2}\right)-\frac{2}{\beta e^{*} V}\right],
\end{aligned}
$$

where the impinging current is given by $I_{\mathrm{imp}}=\left(e^{2} / h\right) V$, and the experimental voltage-dependent transmission probability $\mathcal{T}_{\text {exp }}$ is defined as the ratio between the transmitted current and the impinging one, $\mathcal{T}_{\exp }=I / I_{\text {imp }}$. By fitting Eq. (61) to the experimental data, an effective charge $e^{*}=e$ was found 


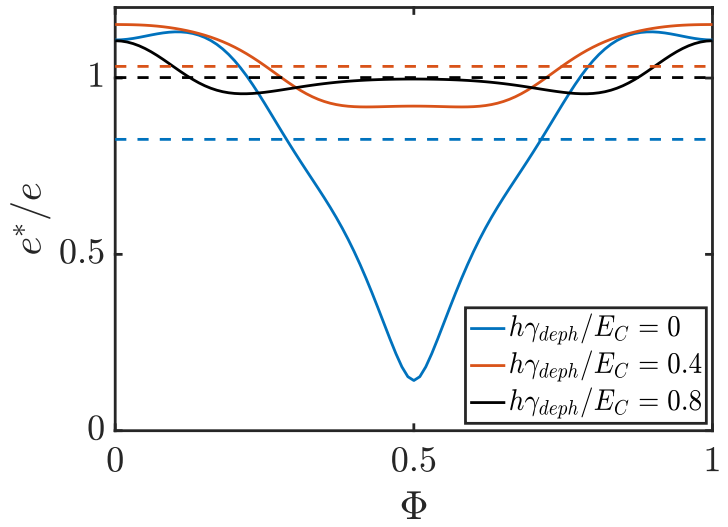

FIG. 12. Effective charge obtained from Eq. (61) as a function of the magnetic flux for a FPI at $v=1$ in the fully coherent case $\left(\gamma_{\text {deph }}=0\right)$ and in presence of dephasing $\left(h \gamma_{\text {deph }} / E_{C}=0.4,0.8\right)$. The horizontal lines indicate the average value of the effective charge. The average effective charge is equal to one electron, $\left\langle e^{*}\right\rangle \approx e$, only if the dephasing is included. Parameters are $\beta E_{C}=20, \mathrm{eV} / E_{C}=0.5$, and $\left|t_{\mathrm{QPC}}\right|^{2}=0.05$.

at filling factor $v=1$, while the charge of an electron pair $e^{*}=2 e$ was discovered at $v=2[12]$.

In order to understand whether the Fano factors computed in the previous sections can be related to an effective charge, we apply the same analysis as in Ref. [12] to the predictions for voltage-dependent current and excess noise computed from Eqs. (56) and (58), treating them as "synthetic" experimental data. In Fig. 12, we display the effective charge $e^{*}$ as a function of the flux for an FPI at $v=1$ (curve $h \gamma_{\text {deph }} / E_{C}=0$ ), and we indicate the flux-averaged effective charge $\left\langle e^{*}\right\rangle$ with the dashed line. We see that in the closed limit considered here the effective charge significantly varies as a function of flux and that its flux-averaged value is less than one. Clearly, the tunneling object is always one electron, and the effective charge $e^{*}<1$ is a manifestation of interaction effects combined with the Pauli principle.

In experimental systems, the electrons traveling along the interferometer do not completely preserve phase coherence but are subject to dephasing. To model such dephasing, we assume that the interfering electrons are subject to a slowly varying potential that causes the energy of the electron to fluctuate,

$$
E_{\mathrm{deph}}=E+\delta E,
$$

where $E$ is the fixed part, while $\delta E$ represents the fluctuating part of the energy. In the following, we parametrize the fluctuations as

$$
\delta E=h \gamma_{\mathrm{deph}} \varphi,
$$

where $\varphi \in[-0.5,0.5]$ is a random variable and $\gamma_{\text {deph }}$ provides the amount of dephasing. If $\gamma_{\mathrm{deph}}=0$ the interferometer is fully coherent, while the totally dephased limit is obtained for $h \gamma_{\mathrm{deph}}=E_{C}$. The effect of dephasing is to reduce the maximum of the transmission probability, and increase the width of transmission resonances. In Fig. 13, we show the conductance as a function of the magnetic flux in the presence of dephasing. The amplitude of the oscillations of the conductance is smaller for stronger dephasing, resulting in

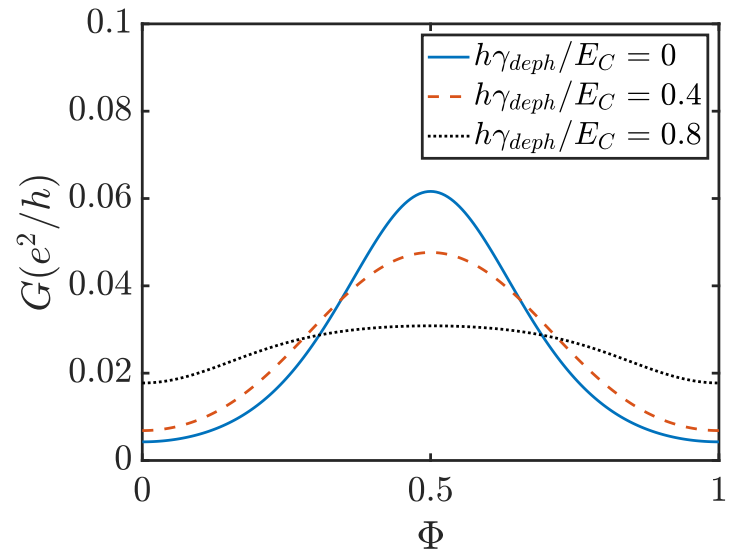

FIG. 13. Conductance as a function of the magnetic flux for a FPI at $v=1$ without $\left(\gamma_{\text {deph }}=0\right)$ and with $\left(h \gamma_{\text {deph }} / E_{C}=0.4,0.8\right)$ dephasing for $\beta E_{C}=10,\left|t_{\mathrm{QPC}}\right|^{2}=0.05$. The fully dephased interferometer would correspond to $h \gamma_{\text {deph }}=E_{C}$.

a reduction of the interference visibility. We next generate "synthetic" experimental data by averaging the transmission probability Eq. (52) over energy according to Eq. (63). We then compute noise and Fano factor (see Appendix B for details). We display the effect of the dephasing on the Fano factor in Fig. 14. It is evident that the dephasing reduces the oscillation amplitude of the Fano factor. In addition, we note that the Fano factor is always smaller than one for the chosen parameters. Finally, we compute an effective charge by fitting our synthetic data with Eq. (61). The value of the effective charge is plotted as a function of the magnetic flux in Fig. 12 for different values of $\gamma_{\text {deph }}$. We indicate the respective average values with the dashed lines. We find that the inclusion of dephasing reduces the flux dependence of the effective charge, making it more meaningful to take the flux average of the effective charge. By averaging over the flux, we obtain $\left\langle e^{*}\right\rangle \approx e$ when the dephasing is included. We conclude that incorporating a dephasing mechanism into the description of a relatively closed FPI is crucial to obtaining a meaningful effective charge. Since the interplay of

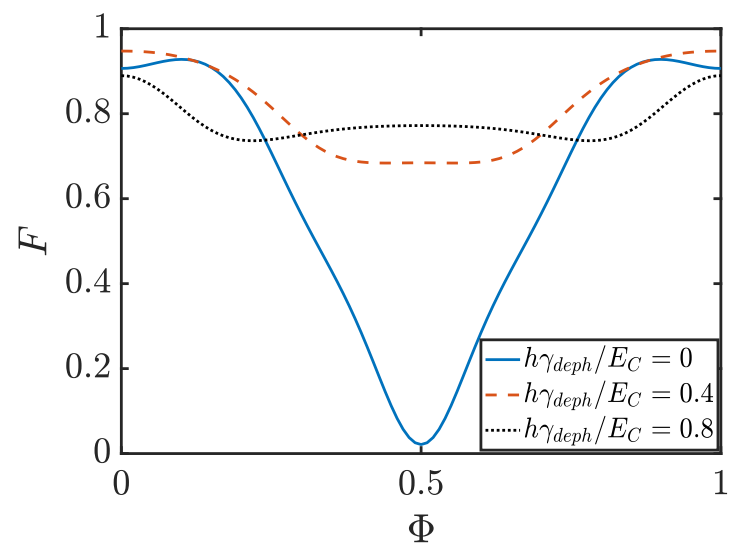

FIG. 14. Fano factor as a function of the magnetic flux for a FPI at $v=1$ without $\left(\gamma_{\text {deph }}=0\right)$ and with $\left(h \gamma_{\text {deph }} / E_{C}=0.4,0.8\right)$ dephasing. The parameters are $\beta E_{C}=20, \mathrm{eV} / E_{C}=0.5$, and $|t|^{2}=0.05$ for all the plots. 
interactions and dephasing in the framework of the master equation formalism is beyond the scope of the present work, we do not present the results of our shot noise calculations in terms of an effective charge $e^{*}$. Instead, we rather focus on the relative enhancement of the Fano factor between the case $v=2$ and $v=1$, due to excitation of neutralons.

\section{CONCLUSIONS}

In this work, we have studied an integer quantum Hall interferometer with interedge coupling in the strong backscattering limit. By means of a master equation analysis, we have computed both conductance and noise of the system. We have found that in the presence of a strong repulsive interedge interaction, neutral plasmons contribute to the electronic transport, leading to a noticeable enhancement of excess noise in a nonequilibrium situation. In the limit of low temperature and strong interedge coupling, we have found a doubling of the Fano factor relative to the noninteracting case, indicative of correlated transmission of electrons through the interferometer. We have interpreted this enhancement of noise in terms of an electron attraction mechanism mediated by neutral plasmons and have argued that our results for interferometers in the strong backscattering limit are related to an enhancement of shot noise observed experimentally in more open devices.

\section{ACKNOWLEDGMENTS}

We would like to thank B. I. Halperin and M. Heiblum for helpful discussions. This work was supported by the IMPRS MiS Leipzig and by DFG Grant No. RO 2247/11-1.

\section{APPENDIX A: CALCULATION OF MATRIX ELEMENT}

We provide here some more details about the calculation of the matrix element for the tunneling of one electron in a FPI. The fermionic operator $\psi_{1, D}(x)$, responsible for the annihilation of one electron in the interfering edge of the dot at position $x$, has the form [41]

$$
\psi_{1, D}(x)=\frac{1}{\sqrt{2 \pi \alpha}} e^{-i \chi_{1}} e^{2 \pi i N_{1} x / L} e^{i \phi_{1, D}^{p}(x)}=\frac{1}{\sqrt{2 \pi \alpha}} e^{-i \chi_{1}} e^{2 \pi i N_{1} x / L} e^{i\left(\phi_{\rho}^{p}(x)-\phi_{\sigma}^{p}(x)\right) / \sqrt{2}} .
$$

Here the cutoff $\alpha$ is on the order of the magnetic length, and the associated energy scale is the cyclotron or exchange gap. Since we are interested in low-energy and long-distance physics as compared to these scales, we can send $\alpha$ to zero at the end of the calculation of the matrix element. The needed matrix element is

$$
\begin{aligned}
& \left\langle N_{1}, N_{2},\{n\}_{\rho},\{n\}_{\sigma}\left|\psi_{1, D}(x)\right| N+1, N_{2},\left\{n^{\prime}\right\}_{\rho},\left\{n^{\prime}\right\}_{\sigma}\right\rangle \\
& \quad=\frac{1}{\sqrt{2 \pi \alpha}}\left\langle N_{1}\left|e^{-i \chi_{1}} e^{2 \pi i N_{1} x / L}\right| N_{1}+1\right\rangle\left\langle\{n\}_{\rho}\left|e^{i \phi_{\rho}^{p}(x) / \sqrt{2}}\right|\left\{n^{\prime}\right\}_{\rho}\right\rangle\left\langle\{n\}_{\sigma}\left|e^{-i \phi_{\sigma}^{p}(x) / \sqrt{2}}\right|\left\{n^{\prime}\right\}_{\sigma}\right\rangle \\
& \quad=\frac{c}{\sqrt{2 \pi \alpha}} e^{2 \pi i\left(N_{1}+1\right) x / L}\left\langle\{n\}_{\rho}\left|e^{i \phi_{\rho}^{p}(x) / \sqrt{2}}\right|\left\{n^{\prime}\right\}_{\rho}\right\rangle\left\langle\{n\}_{\sigma}\left|e^{-i \phi_{\sigma}^{p}(x) / \sqrt{2}}\right|\left\{n^{\prime}\right\}_{\sigma}\right\rangle,
\end{aligned}
$$

where $c$ is a phase factor, $|c|^{2}=1$, and we used that $e^{-i \chi}|N+1\rangle=c|N\rangle$, that follows from the commutation relation between $\chi$ and $N,[\chi, N]=i$. The matrix element for plasmon excitations is

$$
\left\langle\{n\}\left|e^{i \phi^{p}(x) / \sqrt{2}}\right|\left\{n^{\prime}\right\}\right\rangle=\left\langle\{n\}\left|e^{i \sum_{k>0}\left(\beta_{k} b_{k}+\beta_{k}^{*} b_{k}^{\dagger}\right)}\right|\left\{n^{\prime}\right\}\right\rangle=\prod_{k>0}\left\langle n_{k}\left|e^{i\left(\beta_{k} b_{k}+\beta_{k}^{*} b_{k}^{\dagger}\right)}\right| n_{k}^{\prime}\right\rangle,
$$

where $b_{k}, b_{k}^{\dagger}$ are bosonic operators satisfying $\left[b_{k}, b_{q}\right]=0,\left[b_{k}, b_{q}^{\dagger}\right]=\delta_{k, q}$, and we defined

$$
\beta_{k}=\sqrt{\frac{\pi}{k L}} e^{i k x-\alpha k / 2}
$$

Now, we have for $n_{k}>n_{k}^{\prime}$

$$
\begin{aligned}
\left\langle n_{k}\left|e^{i\left(\beta_{k} b_{k}+\beta_{k}^{*} b_{k}^{\dagger}\right)}\right| n_{k}^{\prime}\right\rangle & =e^{\left|\beta_{k}\right|^{2} / 2}\left\langle n_{k}\left|e^{i \beta_{k} b_{k}} e^{i \beta_{k}^{*} b_{k}^{\dagger}}\right| n_{k}^{\prime}\right\rangle \\
& =e^{\left|\beta_{k}\right|^{2} / 2} \sum_{l, m} \frac{\left(i \beta_{k}\right)^{l}}{l !} \frac{\left(i \beta_{k}^{*}\right)^{m}}{m !}\left\langle n_{k}\left|b_{k}^{l}\left(b_{k}^{\dagger}\right)^{m}\right| n_{k}^{\prime}\right\rangle \\
& =e^{\left|\beta_{k}\right|^{2} / 2} \sum_{l, m} \frac{\left(i \beta_{k}\right)^{l}}{l !} \frac{\left(i \beta_{k}^{*}\right)^{m}}{m !} \frac{1}{\sqrt{n_{k} ! n_{k}^{\prime} !}}\left\langle 0\left|b_{k}^{\left(l+n_{k}\right)}\left(b_{k}^{\dagger}\right)^{\left(m+n_{k}^{\prime}\right)}\right| 0\right\rangle \\
& =e^{\left|\beta_{k}\right|^{2} / 2} \sum_{l, m} \frac{\left(i \beta_{k}\right)^{l}}{l !} \frac{\left(i \beta_{k}^{*}\right)^{m}}{m !} \sqrt{\frac{\left(l+n_{k}\right) !\left(m+n_{k}^{\prime}\right) !}{n_{k} ! n_{k}^{\prime} !}}\left\langle l+n_{k} \mid m+n_{k}^{\prime}\right\rangle
\end{aligned}
$$




$$
\begin{aligned}
& =e^{\left|\beta_{k}\right|^{2} / 2} \sum_{l} \frac{\left(i \beta_{k}\right)^{l}}{l !} \frac{\left(i \beta_{k}^{*}\right)^{l+n_{k}-n_{k}^{\prime}}}{\left(l+n_{k}-n_{k}^{\prime}\right) !} \frac{\left(l+n_{k}\right) !}{\sqrt{n_{k} ! n_{k}^{\prime} !}} \\
& =e^{\left|\beta_{k}\right|^{2} / 2} \frac{\left(i \beta_{k}^{*}\right)^{n_{k}-n_{k}^{\prime}}}{\sqrt{n_{k} ! n_{k}^{\prime} !}} \sum_{l} \frac{\left(l+n_{k}\right) !}{l !\left(l+n_{k}-n_{k}^{\prime}\right) !}\left(-\left|\beta_{k}\right|^{2}\right)^{l} \\
& =e^{\left|\beta_{k}\right|^{2} / 2} \frac{\left(i \beta_{k}^{*}\right)^{n_{k}-n_{k}^{\prime}}}{\left(n_{k}-n_{k}^{\prime}\right) !} \sqrt{\frac{n_{k} !}{n_{k}^{\prime} !}} \Phi\left(n_{k}+1, n_{k}-n_{k}^{\prime}+1,-\left|\beta_{k}\right|^{2}\right) \\
& =e^{-\left|\beta_{k}\right|^{2} / 2}\left(i \beta_{k}^{*}\right)^{n_{k}-n_{k}^{\prime}} \sqrt{\frac{n_{k}^{\prime} !}{n_{k} !}} L_{n_{k}^{\prime}}^{n_{k}-n_{k}^{\prime}}\left(\left|\beta_{k}\right|^{2}\right),
\end{aligned}
$$

where we used the Baker-Campbell-Hausdorff formula, the power series of the exponential, the orthonormal states

$$
\left|n_{k}\right\rangle=\frac{\left(b_{k}^{\dagger}\right)^{n_{k}}}{\sqrt{n_{k} !}}|0\rangle
$$

the definition of the confluent hypergeometric function $\Phi$, and its relation to the associated Laguerre polynomials $L_{a}^{b}(x)$

$$
\Phi\left(n_{k}+1, n_{k}-n_{k}^{\prime}+1,-\left|\beta_{k}\right|^{2}\right)=\frac{\left(n_{k}-n_{k}^{\prime}\right) !}{n_{k} !} \sum_{l} \frac{\left(l+n_{k}\right) !}{l !\left(l+n_{k}-n_{k}^{\prime}\right) !}\left(-\left|\beta_{k}\right|^{2}\right)^{l}=\frac{n_{k}^{\prime} !\left(n-n_{k}^{\prime}\right) !}{n_{k} !} e^{-\left|\beta_{k}\right|^{2}} L_{n_{k}^{\prime}}^{n_{k}-n_{k}^{\prime}}\left(\left|\beta_{k}\right|^{2}\right) .
$$

By inserting Eq. (A5) in Eq. (A3), taking the modulus square, and using Eq. (A4) and the momentum quantization $k=2 \pi m / L$, we get

$$
\begin{aligned}
\left|\left\langle\{n\}\left|e^{i \phi^{p}(x) / \sqrt{2}}\right|\left\{n^{\prime}\right\}\right\rangle\right|^{2} & =\prod_{k>0} e^{-\left|\beta_{k}\right|^{2}}\left|\beta_{k}\right|^{2\left|n_{k}-n_{k}^{\prime}\right|}\left(\frac{n_{k}^{(<)} !}{n_{k}^{(>)} !}\right)\left[L_{n_{k}^{(<)}}^{\left|n_{k}-n_{k}^{\prime}\right|}\left(\left|\beta_{k}\right|^{2}\right)\right]^{2} \\
& =\prod_{m=1}^{+\infty} e^{-(1 / 2 m) e^{-2 \pi \alpha m / L}}\left(\frac{1}{2 m} e^{-2 \pi \alpha m / L}\right)^{\left|n_{m}-n_{m}^{\prime}\right|}\left(\frac{n_{m}^{(<)} !}{n_{m}^{(>)} !}\right)\left[L_{n_{m}^{(<)}}^{\left|n_{m}-n_{m}^{\prime}\right|}\left(\frac{1}{2 m} e^{-2 \pi \alpha m / L}\right)\right]^{2} .
\end{aligned}
$$

For implementing the rate equation formalism numerically, we need to truncate the Hilbert space to a finite size and let $n_{m}=n_{m}^{\prime}=0$ for every $m>m_{\max }$. Since the excitation of high-energy plasmons is unlikely due to the exponential cutoff in the Fermi distribution, we choose $m_{\max }$ as the smallest integer number satisfying $m_{\max }>\left(\mathrm{eV}+8 k_{B} T\right) / E_{\sigma}$. Then, we can write Eq. (A8) as

$$
\begin{aligned}
& \left|\left\langle\{n\}\left|e^{i \phi^{p}(x) / \sqrt{2}}\right|\left\{n^{\prime}\right\}\right\rangle\right|^{2} \\
& =\prod_{m=1}^{m_{\max }} e^{-(1 / 2 m) e^{-2 \pi \alpha m / L}}\left(\frac{1}{2 m} e^{-2 \pi \alpha m / L}\right)^{\left|n_{m}-n_{m}^{\prime}\right|} \frac{n_{m}^{(<)} !}{n_{m}^{(>)} !}\left[L_{n_{m}^{(<)}}^{\left|n_{m}-n_{m}^{\prime}\right|}\left(\frac{1}{2 m} e^{-2 \pi \alpha m / L}\right)\right]^{2} \prod_{m>m_{\max }} e^{-(1 / 2 m) e^{-2 \pi \alpha m / L}} \\
& =\prod_{m=1}^{m_{\max }} e^{-(1 / 2 m) e^{-2 \pi \alpha m / L}}\left(\frac{1}{2 m} e^{-2 \pi \alpha m / L}\right)^{\left|n_{m}-n_{m}^{\prime}\right|} \frac{n_{m}^{(<)} !}{n_{m}^{(>)} !}\left[L_{n_{m}^{(<)}}^{\left|n_{m}-n_{m}^{\prime}\right|}\left(\frac{1}{2 m} e^{-2 \pi \alpha m / L}\right)\right]^{2} \frac{\prod_{m=1}^{+\infty} e^{-(1 / 2 m) e^{-2 \pi \alpha m / L}}}{\prod_{m=1}^{m_{\max }} e^{-(1 / 2 m) e^{-2 \pi \alpha m / L}}} \\
& =\sqrt{1-e^{-2 \pi \alpha / L}} \frac{\prod_{m=1}^{m_{\max }} e^{-(1 / 2 m) e^{-2 \pi \alpha m / L}}\left(\frac{1}{2 m} e^{-2 \pi \alpha / L}\right)^{\left|n_{m}-n_{m}^{\prime}\right|} \frac{n_{m}^{(\times) !}}{n_{m}^{(\otimes) !}}\left[L_{n_{m}^{(<)}}^{\left|n_{m}-n_{m}^{\prime}\right|}\left(\frac{1}{2 m} e^{-2 \pi \alpha m / L}\right)\right]^{2}}{\prod_{m=1}^{m_{\max }} e^{-(1 / 2 m) e^{-2 \pi \alpha m / L}}},
\end{aligned}
$$

and we obtain the final result by inserting Eq. (A9) into Eq. (A2) and taking the limit $\alpha \rightarrow 0$.

\section{APPENDIX B: EFFECTIVE CHARGE AND DEPHASING}

In this Appendix, we provide some more details about the inclusion of the dephasing in extracting an effective charge from excess noise. First, we show in Fig. 15 a comparison between the values for effective charges at $v=1$ in the closed and open limits. The flux-averaged effective charge is indicated with the dashed line. In the closed limit, the effective charge strongly oscillates with the flux. On the other hand, the oscillations of $e^{*}$ are much less pronounced in the open limit, and we find the expected effective charge $e^{*} \approx e$. Therefore, the standard procedure in Eq. (61) to extract the effective charge from the shot noise works well in the open limit but does not give useful results when the interferometer operates in the closed limit. For this reason, we do not convert our predictions for excess noise at $v=2$ into effective charges.

When including dephasing into the description of the FPI, an effective charge can be extracted for interferometers operating in the closed limit as well. According to Eq. (63), the 


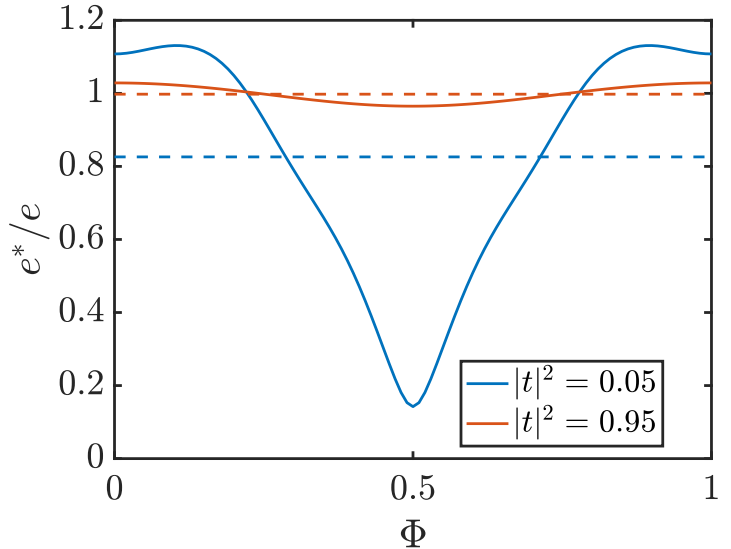

FIG. 15. Effective charge as a function of the magnetic flux obtained from Eq. (61) in the closed limit $\left(|t|^{2}=0.05\right)$ and in the open limit $\left(|t|^{2}=0.95\right)$ at voltage $\mathrm{eV} / E_{C}=0.5$ and temperature $\beta E_{C}=20$. The dashed lines represent the flux-averaged effective charge.

transmission probability for a FPI subject to the dephasing is

$$
\mathcal{T}\left(E, \gamma_{\mathrm{deph}}, \varphi\right)=\frac{|t|^{4}}{\left|1+r^{2} e^{2 \pi i\left(\frac{E}{E_{C}}+\Phi+\frac{h \gamma_{\mathrm{deph}}}{E_{C}} \varphi\right)}\right|^{2}},
$$

and by averaging over the random variable $\varphi$ we have

$$
\mathcal{T}\left(E, \gamma_{\text {deph }}\right)=\int_{-0.5}^{0.5} d \varphi \mathcal{T}\left(E, \gamma_{\text {deph }}, \varphi\right) .
$$

The conductance is obtained from Eq. (B2) as

$$
G=\frac{e^{2}}{h} \int d E \mathcal{T}\left(E, \gamma_{\text {deph }}\right)\left(-\frac{\partial f}{\partial E}\right) .
$$

To quantify the quality of the interference signal, we define the visibility $\mathcal{V}$ as

$$
\mathcal{V}=\frac{\max (G)-\min (G)}{\max (G)+\min (G)} .
$$

We plot the visibility as a function of temperature for different values of $\gamma_{\text {deph }}$ in the right panel of Fig. 16. We can immediately see that the presence of dephasing strongly reduces the visibility. In the absence of dephasing, the visibility decays exponentially with temperature (see Fig. 16), while in the presence of dephasing deviations from an exponential decay are visible. Overall, the visibility decays faster when the dephasing becomes stronger.

We now want to calculate the excess noise in the presence of the dephasing modeled in Eqs. (62) and (63). In the main text, we first averaged the transmission probability $\mathcal{T}\left(E, \gamma_{\text {deph }}, \varphi\right)$ over $\varphi$ and then calculate the noise from it. Therefore, the dephased noise is similar to Eq. (58) but with the transmission probability given by Eq. (B2):

$$
\begin{aligned}
S= & \frac{2 e^{2}}{h} \int d E\left\{\mathcal{T}\left(E, \gamma_{\text {deph }}\right)\left[f_{L}\left(1-f_{L}\right)+f_{R}\left(1-f_{R}\right)\right]\right. \\
& \left.+\mathcal{T}\left(E, \gamma_{\text {deph }}\right)\left[1-\mathcal{T}\left(E, \gamma_{\text {deph }}\right)\right]\left(f_{L}-f_{R}\right)^{2}\right\} .
\end{aligned}
$$

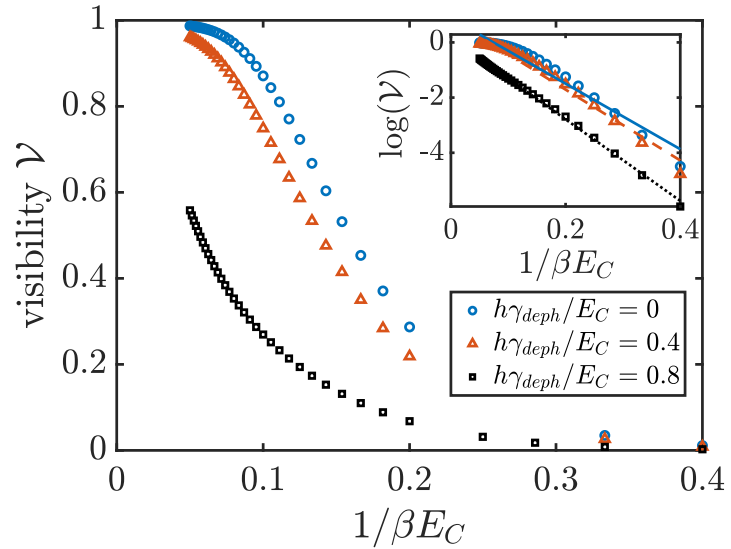

FIG. 16. Visibility, defined in Eq. (B4), of a FPI at $v=1$ as a function of the temperature for different values of $\gamma_{\text {deph }}$. In the inset, the logarithm of the visibility is plotted as a function of the temperature.

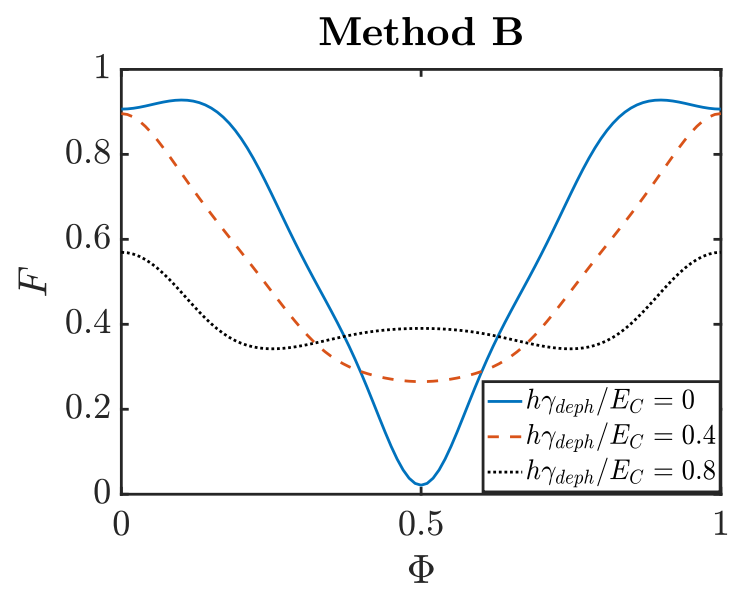

FIG. 17. Fano factor as a function of the magnetic flux in the presence of dephasing $\left(h \gamma_{\mathrm{deph}} / E_{C}=0.4,0.8\right)$, calculated from Eq. (B6). The parameters are $\beta E_{C}=20, \mathrm{eV} / E_{C}=0.5$, and $|t|^{2}=$ 0.05 .

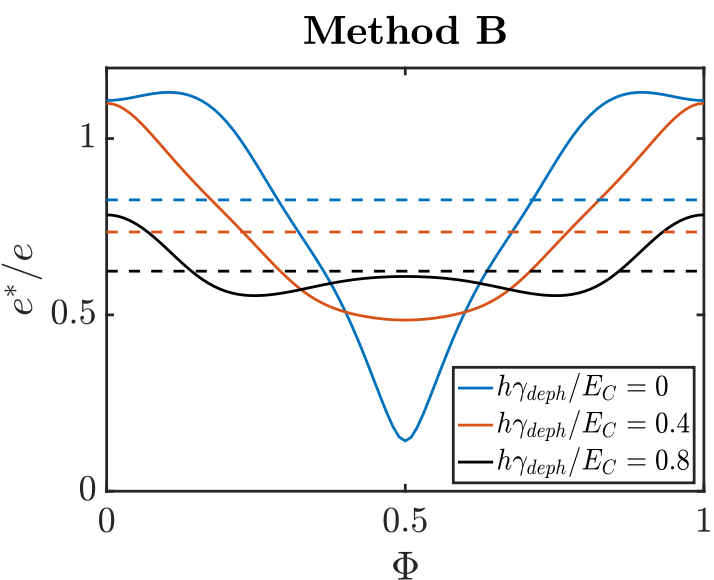

FIG. 18. Effective charge extracted from the noise calculated from Eq. (B6). The horizontal lines indicate the average value of the effective charge. The effective charge is reduced when the dephasing is stronger. Parameters are $\beta E_{C}=20, \mathrm{eV} / E_{C}=0.5$, and $\left|t_{\mathrm{QPC}}\right|^{2}=0.05$. 
The Fano factor obtained from Eq. (B5) is shown in Fig. 14 and the effect of the dephasing is to diminish the amplitude of its oscillations.

We now discuss a different method (method B) to calculate the noise in presence of dephasing. This consists in calculating the noise for a given $\varphi$ and just at the end the average over $\varphi$ is performed. Accordingly, the noise is given by

$$
\begin{aligned}
S= & \frac{2 e^{2}}{h} \int d \varphi \int d E\left\{\mathcal{T}\left(E, \gamma_{\mathrm{deph}}, \varphi\right)\right. \\
& \times\left[f_{L}\left(1-f_{L}\right)+f_{R}\left(1-f_{R}\right)\right] \\
& \left.+\mathcal{T}\left(E, \gamma_{\mathrm{deph}}, \varphi\right)\left[1-\mathcal{T}\left(E, \gamma_{\mathrm{deph}}, \varphi\right)\right]\left(f_{L}-f_{R}\right)^{2}\right\},
\end{aligned}
$$

with $\mathcal{T}\left(E, \gamma_{\text {deph }}, \varphi\right)$ given by Eq. (B1). Equation (B6) corresponds to averaging the nondephased noise in Eq. (58) over the flux $\Phi$ in a window of length $h \gamma_{\text {deph }} / E_{C}$, as it can be seen from Eq. (B1) and the change of variable $\Phi^{\prime}=\Phi+$ $\left(h \gamma_{\mathrm{deph}} / E_{C}\right) \varphi$. This way of including the dephasing would be directly applicable also to the master equation. Accordingly, the Fano factor obtained from Eq. (B6) is shown in Fig. 17. As an effect of the dephasing, the amplitude of the oscillations of $F$ gets smaller.

Finally, we compute the effective charge by using Eq. (61), as in Ref. [12], from the noise calculated with the two different methods. In the main text (see Fig. 12), we have shown the effective charge obtained from the noise calculated with Eq. (B5), and we have seen that the dephasing helps to get $\left\langle e^{*}\right\rangle=e$. The effective charge is plotted as a function of the magnetic flux in Fig. 18 for method B and different values of $\gamma_{\text {deph }}$. We indicate the respective average values with the dashed lines. We find that in contrast to the results in Fig. 12, the average effective charge $\left\langle e^{*}\right\rangle$ gets smaller for stronger dephasing, when Eq. (B6) is used to obtain the noise. Therefore, Eqs. (61) and (B6) are not compatible with each other. Since method B is not helpful in extracting an effective charge with a value close to the electron charge in the test case $v=1$, we do not attempt to compute an effective charge in the more complicated case $v=2$, and rather present the relative enhancement of the Fano factor as our main result.
[1] B. I. Halperin, Statistics of Quasiparticles and the Hierarchy of Fractional Quantized Hall States, Phys. Rev. Lett. 52, 1583 (1984).

[2] D. Arovas, J. R. Schrieffer, and F. Wilczek, Fractional Statistics and the Quantum Hall Effect, Phys. Rev. Lett. 53, 722 (1984).

[3] A. Stern, Anyons and the quantum Hall effect: A pedagogical review, Ann. Phys. 323, 204 (2008).

[4] C. de C. Chamon, D. E. Freed, S. A. Kivelson, S. L. Sondhi, and X. G. Wen, Two point-contact interferometer for quantum Hall systems, Phys. Rev. B 55, 2331 (1997).

[5] F. E. Camino, W. Zhou, and V. J. Goldman, Aharonov-Bohm electron interferometer in the integer quantum Hall regime, Phys. Rev. B 72, 155313 (2005).

[6] F. E. Camino, W. Zhou, and V. J. Goldman, Quantum transport in electron Fabry-Perot interferometers, Phys. Rev. B 76, 155305 (2007).

[7] D. T. McClure, Y. Zhang, B. Rosenow, E. M. Levenson-Falk, C. M. Marcus, L. N. Pfeiffer, and K. W. West, Edge-State Velocity and Coherence in a Quantum Hall Fabry-Pérot Interferometer, Phys. Rev. Lett. 103, 206806 (2009).

[8] H. Choi, P.-h. Jiang, M. Godfrey, W. Kang, S. Simon, L. Pfeiffer, K. West, and K. Baldwin, Aharonov-Bohm-like oscillations in Fabry-Perot interferometers, New J. Phys. 13, 055007 (2011).

[9] Y. Zhang, D. T. McClure, E. M. Levenson-Falk, C. M. Marcus, L. N. Pfeiffer, and K. W. West, Distinct signatures for Coulomb blockade and Aharonov-Bohm interference in electronic FabryPerot interferometers, Phys. Rev. B 79, 241304(R) (2009).

[10] N. Ofek, A. Bid, M. Heiblum, A. Stern, V. Umansky, and D. Mahalu, Role of interactions in an electronic Fabry-Perot interferometer operating in the quantum Hall effect regime, Proc. Natl. Acad. Sci. USA 107, 5276 (2010).

[11] S. Baer, C. Rössler, T. Ihn, K. Ensslin, C. Reichl, and W. Wegscheider, Cyclic depopulation of edge states in a large quantum dot, New J. Phys. 15, 023035 (2013).
[12] H. Choi, I. Sivan, A. Rosenblatt, M. Heiblum, V. Umansky, and D. Mahalu, Robust electron pairing in the integer quantum Hall effect regime, Nat. Commun. 6, 7435 (2015).

[13] I. Sivan, H. Choi, J. Park, A. Rosenblatt, Y. Gefen, D. Mahalu, and V. Umansky, Observation of interaction-induced modulations of a quantum Hall liquid's area, Nat. Commun. 7, 12184 (2016).

[14] I. Sivan, R. Bhattacharyya, H. K. Choi, M. Heiblum, D. E. Feldman, D. Mahalu, and V. Umansky, Interaction-induced interference in the integer quantum Hall effect, Phys. Rev. B 97, 125405 (2018).

[15] J. Nakamura, S. Fallahi, H. Sahasrabudhe, R. Rahman, S. Liang, G. C. Gardner, and M. J. Manfra, Aharonov-Bohm interference of fractional quantum Hall edge modes, Nat. Phys. 15, 563 (2019).

[16] M. P. Röösli, L. Brem, B. Kratochwil, G. Nicolí, B. A. Braem, S. Hennel, P. Märki, M. Berl, C. Reichl, W. Wegscheider, K. Ensslin, T. Ihn, and B. Rosenow, Observation of quantum Hall interferometer phase jumps due to a change in the number of bulk quasiparticles, Phys. Rev. B 101, 125302 (2020).

[17] J. Nakamura, S. Liang, G. C. Gardner, and M. J. Manfra, Direct observation of anyonic braiding statistics, Nat. Phys. 16, 931 (2020).

[18] B. Rosenow and B. I. Halperin, Influence of Interactions on Flux and Back-Gate Period of Quantum Hall Interferometers, Phys. Rev. Lett. 98, 106801 (2007).

[19] B. I. Halperin, A. Stern, I. Neder, and B. Rosenow, Theory of the Fabry-Pérot quantum Hall interferometer, Phys. Rev. B 83, 155440 (2011).

[20] S. Ngo Dinh and D. A. Bagrets, Influence of Coulomb interaction on the Aharonov-Bohm effect in an electronic Fabry-Pérot interferometer, Phys. Rev. B 85, 073403 (2012).

[21] A. Kamenev and Y. Gefen, Coulomb Blockade with Neutral Modes, Phys. Rev. Lett. 114, 156401 (2015). 
[22] G. A. Frigeri, D. D. Scherer, and B. Rosenow, Sub-periods and apparent pairing in integer quantum Hall interferometers, Europhys. Lett. 126, 67007 (2019).

[23] C. Nayak, S. H. Simon, A. Stern, M. Freedman, and S. Das Sarma, Non-Abelian anyons and topological quantum computation, Rev. Mod. Phys. 80, 1083 (2008).

[24] D. Ferraro and E. Sukhorukov, Interaction effects in a multichannel Fabry-Pérot interferometer in the Aharonov-Bohm regime, SciPost Phys. 3, 014 (2017).

[25] B. Keimer, S. A. Kivelson, M. R. Norman, S. Uchida, and J. Zaanen, From quantum matter to high-temperature superconductivity in copper oxides, Nature (London) 518, 179 (2015).

[26] M. E. Raikh, L. I. Glazman, and L. E. Zhukov, Two-Electron State in a Disordered 2D Island: Pairing Caused by the Coulomb Repulsion, Phys. Rev. Lett. 77, 1354 (1996).

[27] A. Hamo, A. Benyamini, I. Shapir, I. Khivrich, J. Waissman, K. Kaasbjerg, Y. Oreg, F. von Oppen, and S. Ilani, Electron attraction mediated by Coulomb repulsion, Nature (London) 535, 395 (2016).

[28] C. Hong, G. Yoo, J. Park, M.-K. Cho, Y. Chung, H.-S. Sim, D. Kim, H. Choi, V. Umansky, and D. Mahalu, Attractive Coulomb interactions in a triple quantum dot, Phys. Rev. B 97, 241115(R) (2018).

[29] J. I. Väyrynen, M. Goldstein, and Y. Gefen, Superconducting Correlations out of Repulsive Interactions on a Fractional Quantum Hall Edge, Phys. Rev. Lett. 122, 236802 (2019).

[30] A. N. Korotkov, Intrinsic noise of the single-electron transistor, Phys. Rev. B 49, 10381 (1994).

[31] J. U. Kim, I. V. Krive, and J. M. Kinaret, Nonequilibrium Plasmons in a Quantum Wire Single-Electron Transistor, Phys. Rev. Lett. 90, 176401 (2003).

[32] S. S. Safonov, A. K. Savchenko, D. A. Bagrets, O. N. Jouravlev, Y. V. Nazarov, E. H. Linfield, and D. A. Ritchie, Enhanced Shot Noise in Resonant Tunneling Via Interacting Localized States, Phys. Rev. Lett. 91, 136801 (2003).

[33] W. Belzig, Full counting statistics of super-Poissonian shot noise in multilevel quantum dots, Phys. Rev. B 71, 161301(R) (2005).

[34] A. Carmi and Y. Oreg, Enhanced shot noise in asymmetric interacting two-level systems, Phys. Rev. B 85, 045325 (2012).

[35] A. Stern, B. Rosenow, R. Ilan, and B. I. Halperin, Interference, Coulomb blockade, and the identification of non-Abelian quantum Hall states, Phys. Rev. B 82, 085321 (2010).

[36] J. I. Väyrynen and L. I. Glazman, Current Noise from a Magnetic Moment in a Helical Edge, Phys. Rev. Lett. 118, 106802 (2017).
[37] P. D. Kurilovich, V. D. Kurilovich, I. S. Burmistrov, Y. Gefen, and M. Goldstein, Unrestricted Electron Bunching at the Helical Edge, Phys. Rev. Lett. 123, 056803 (2019).

[38] J. von Delft and H. Schoeller, Bosonization for beginnersrefermionization for experts, Ann. Phys. 7, 225 (1998).

[39] T. Giamarchi, Quantum Physics in One Dimension, International Series of Monographs on Physics, Vol. 121 (Clarendon Press, Oxford, 2003).

[40] J. Dempsey, B. Y. Gelfand, and B. I. Halperin, ElectronElectron Interactions and Spontaneous Spin Polarization in Quantum Hall Edge States, Phys. Rev. Lett. 70, 3639 (1993).

[41] M. R. Geller and D. Loss, Aharonov-Bohm effect in the chiral Luttinger liquid, Phys. Rev. B 56, 9692 (1997).

[42] Y. V. Nazarov and Y. M. Blanter, Quantum Transport: Introduction to Nanoscience (Cambridge University Press, Cambridge, UK, 2009).

[43] M. Abramowitz and I. A. Stegun, Orthogonal polynomials, in Handbook of Mathematical Functions: With Formulas, Graphs, and Mathematical Tables (Dover, New York, 1972), pp. 771802.

[44] J. U. Kim, M.-S. Choi, I. V. Krive, and J. M. Kinaret, Nonequilibrium plasmons and transport properties of a double-junction quantum wire, Low Temp. Phys. 32, 1158 (2006).

[45] A. Furusaki, Resonant tunneling through a quantum dot weakly coupled to quantum wires or quantum Hall edge states, Phys. Rev. B 57, 7141 (1998).

[46] Y. M. Blanter and M. Büttiker, Shot noise in mesoscopic conductors, Phys. Rep. 336, 1 (2000).

[47] L. DiCarlo, J. R. Williams, Y. Zhang, D. T. McClure, and C. M. Marcus, Shot Noise in Graphene, Phys. Rev. Lett. 100, 156801 (2008).

[48] M. Büttiker, Scattering Theory of Thermal and Excess Noise in Open Conductors, Phys. Rev. Lett. 65, 2901 (1990).

[49] M. Büttiker, Scattering theory of current and intensity noise correlations in conductors and wave guides, Phys. Rev. B 46 , 12485 (1992).

[50] Y. Aharonov and D. Bohm, Significance of electromagnetic potentials in the quantum theory, Phys. Rev. 115, 485 (1959).

[51] T. Ihn, Semiconductor Nanostructures: Quantum States and Electronic Transport (Oxford University Press, Oxford, UK, 2010).

[52] M. Heiblum, Quantum shot noise in edge channels, Phys. Status Solidi (b) 243, 3604 (2006).

[53] T. G. Griffiths, E. Comforti, M. Heiblum, A. Stern, and V. Umansky, Evolution of Quasiparticle Charge in the Fractional Quantum Hall Regime, Phys. Rev. Lett. 85, 3918 (2000). 